\title{
In vivo characterization and application of a novel potassium channel-based optogenetic silencer in the healthy and epileptic mouse hippocampus
}

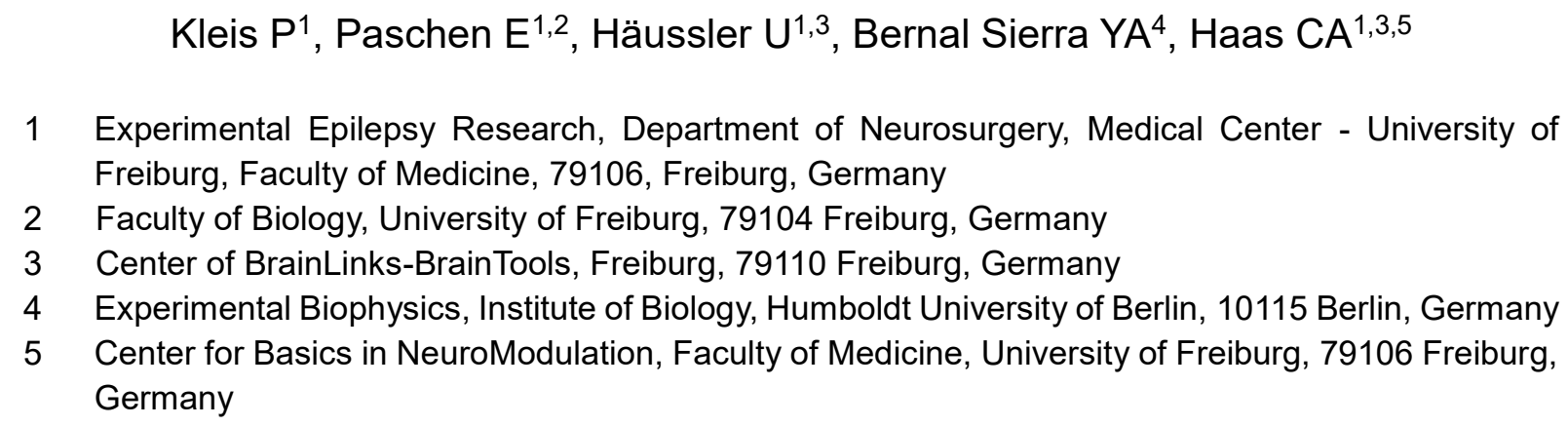

Conflict of interest statement: The authors declare no competing financial interests.

\section{Acknowledgements}

We are grateful to the Viral Core Facility, Charité - Universitätsmedizin Berlin, Germany for providing the viral constructs. We thank Prof. Peter Hegemann and Philipp Janz for intellectual input, Andrea DjieMaletz for excellent technical assistance, Jessica Link for proofreading, and Oana Constantin for unpublished information about the photoactivated adenylyl cyclase, bPAC.

This study was supported by the German Research Foundation (HA 1443/11-1 to CAH, SPP1926 to YABS) and by the BrainLinks-BrainTools Center, which is funded by the Federal Ministry of Economics, Science and Arts of Baden-Württemberg within the sustainability program for projects of the Excellence Initiative II.

Keywords: bPAC, cAMP, electrophysiology, epilepsy, kainate, optogenetic inhibition 


\section{Abstract}

The performance of available optogenetic inhibitors remains insufficient due to low light sensitivity, short-lasting photocurrents, and unintended changes in ion distributions. To overcome these limitations, a novel potassium channel-based optogenetic silencer was developed and successfully applied in various in vitro and acute in vivo settings (Bernal Sierra et al., 2018). This tool, a two-component construct called PACK, comprises a photoactivated adenylyl cyclase (bPAC) and a cAMP-dependent potassium channel (SthK). Here, we examined the long-term inhibitory action and side effects of the PACK construct in healthy and epileptic adult male mice. We targeted hippocampal CA1 pyramidal cells using a viral vector and enabled illumination of these neurons via an implanted optic fiber. Local field potential (LFP) recordings from the CA1 of freely moving mice revealed significantly reduced neuronal activity during $50-$ minute intermittent illumination, especially in the beta and gamma frequency ranges. Adversely, PACK expression in healthy mice induced chronic astrogliosis, dispersion of pyramidal cells, and generalized seizures. These side effects were independent of the light application and were also present in mice expressing bPAC without the potassium channel. Additionally, light-activation of bPAC alone increased neuronal activity, presumably via enhanced cAMP signaling. In chronically epileptic mice, the dark activity of bPAC/PACK in CA1 prevented the spread of spontaneous epileptiform activity from the seizure focus to the contralateral bPAC/PACK-expressing hippocampus. Taken together, the PACK tool is a potent optogenetic inhibitor but requires refinement of its light-sensitive domain to avoid unexpected physiological changes. 


\section{Significance statement}

Optogenetics allows precise manipulation of neuronal activity via genetically encoded light-sensitive proteins. Unfortunately, available optogenetic inhibitors are not suitable for prolonged use. The newly developed two-component potassium channel-based optogenetic inhibitor, PACK, has been identified as a potent silencer of neurons in various acute experiments. Here, we characterized the PACK construct in freely behaving healthy and epileptic mice. We targeted the PACK silencer specifically to CA1 pyramidal neurons, where illumination with short light pulses at low frequencies reliably reduced neuronal activity. In chronically epileptic mice, PACK prevented the spread of epileptiform activity from the seizure focus to the contralateral PACKexpressing hippocampus. The major disadvantage of the PACK silencer is its lightsensitive domain, the bPAC adenylyl cyclase, which may induce side effects.

\section{Introduction}

Cell type-specific inhibition techniques are required in neuroscience to investigate the contribution of neuronal populations in physiological and pathophysiological processes. Optogenetic silencing takes advantage of genetically encoded lightsensitive proteins, allowing to 'switch off' neurons of interest with high temporal and spatial precision. Currently available optogenetic inhibitors such as inward-directed chloride pumps (e.g. halorhodopsins) and outward-directed proton pumps (e.g. archaerhodopsins) have several limitations. Namely, they require continuous highintensity illumination, which can have unexpected excitatory outcomes due to tissue heating, abnormal ion distributions, and strong rebound responses (Wiegert et al., 2017; Owen et al., 2019). Even brief activation of halorhodopsin can change the intracellular chloride concentration, cause a positive shift in $\mathrm{GABA}_{\mathrm{A}}$ receptor reversal 
potential, and decrease the action potential threshold, leading to elevated network excitability (Raimondo et al., 2012; Alfonsa et al., 2015; Sørensen et al., 2017).

Using potassium $\left(\mathrm{K}^{+}\right)$current as the hyperpolarizing factor would be a better approach since the resting state of neurons is based on $\mathrm{K}^{+}$conductance and is thus more physiological than pumping chloride or protons against their electrochemical gradients. Several synthetic light-activated $\mathrm{K}^{+}$channels have been engineered which, however, pose shortcomings such as the requirement of a chemical cofactor (Banghart et al., 2004; Janovjak et al., 2010), utilizing UV light (Kang et al., 2013) or very low photocurrents in mammalian cells (Cosentino et al., 2015). A newly developed $\mathrm{K}^{+}$ channel-based optogenetic silencer could potentially overcome these limitations (Beck et al., 2018; Bernal Sierra et al., 2018). The two-component silencer, named PACK, comprises a soluble photoactivated adenylyl cyclase from the Beggiatoa bacterium (bPAC; Stierl et al., 2011) and a cyclic nucleotide-gated potassium channel from Spirochaeta thermophila (SthK; Brams et al., 2014). The blue light receptor in bPAC activates the cyclase domain thus increasing cytosolic cyclic adenosine monophosphate (cAMP), which subsequently opens the co-expressed SthK channels. Benefits of the PACK silencer include robust expression in mammalian cells, signal amplification, and large long-lasting $\mathrm{K}^{+}$currents. Previously, PACK has been shown to reliably inhibit neuronal firing in cell cultures, acute slice preparations, and anesthetized mice (Bernal Sierra et al., 2018). However, a long-term application in awake mice has so far not been tested. A prolonged precise inhibition technique would be valuable for investigating the contribution of specific cell populations in pathologies such as epilepsy.

Mesial temporal lobe epilepsy (MTLE), the most common type of acquired focal epilepsy in adults, is characterized by spontaneous hippocampal seizures, which are 
105 often pharmacoresistant (Engel J., 2001; Kwan and Sander, 2004). MTLE is usually described as a unilateral disease since the seizures arise in one hemisphere, ipsilateral to the pathological abnormality. However, in some patients and MTLE mouse models, epileptic activity propagates to the contralateral hippocampus (Gloor et al., 1993; Mintzer et al., 2004; Meier et al., 2007; Popovic et al., 2012; Paschen et al., 2020). To

110 scrutinize the performance of PACK and investigate the contribution of the contralateral 111 hippocampus in MTLE, we applied PACK-mediated inhibition in the well-established 112 intrahippocampal kainate (ihpKA) mouse model. This model recapitulates the major 113 pathological features of human MTLE, such as unilateral hippocampal sclerosis with 114 cell loss and gliosis accompanied by subclinical spontaneous seizures (Bouilleret et 115 al., 1999; Riban et al., 2002; Janz et al., 2017). Contralateral CA1 cells in the ihpKA 116 mouse model exhibit elevated activity-related cytoskeleton (Arc) gene expression 117 during the chronic phase of epilepsy (Janz et al., 2018), suggesting that these cells are 118 involved in the contralateral epileptiform activity.

119 Here, we aimed to validate the inhibitory action of the PACK silencer in principal 120 neurons of hippocampal CA1 in freely moving mice. We investigated the long-term 121 histological and electrophysiological effects of the PACK construct and its components 122 (bPAC, AAV9.CaMKII.mCherry) in vivo. We present evidence that PACK activation 123 persistently reduces neuronal activity during illumination with a frequency as low as $124 \quad 0.1 \mathrm{~Hz}$. Furthermore, we applied the PACK silencer in chronically epileptic mice, where 125 PACK-expression in CA1, contralateral to the seizure focus, prevented seizure spread 126 between hemispheres. 


\section{Materials and Methods}

\section{Animals}

Experiments were performed in adult 10 to 21 week-old transgenic male mice (C57BL/6-Tg(Thy1-eGFP)-M-Line) (Feng et al., 2000). For this study, 54 mice were used, each representing an individual experiment. Mice were kept in a $12 \mathrm{~h}$ light/dark cycle at room temperature (RT) with food and water ad libitum. All animal procedures were carried out following the guidelines of the European Community's Council Directive of 22 September 2010 (2010/63/EU) and were approved by the regional council (Regierungspräsidium Freiburg).

\section{Stereotaxic intrahippocampal injections}

Intrahippocampal saline/kainate and recombinant adeno-associated virus (AAV) injections were performed in one surgery in deeply anesthetized mice (ketamine hydrochloride $100 \mathrm{mg} / \mathrm{kg}$, xylazine $5 \mathrm{mg} / \mathrm{kg}$, atropine $0.1 \mathrm{mg} / \mathrm{kg}$ body weight, i.p.) as described previously (Heinrich et al., 2006; Häussler et al., 2012; Janz et al., 2017). Using a Nanoject III (Drummond Scientific Company, Broomall, Pennsylvania, USA), $50 \mathrm{~nL}$ of $0.9 \%$ sterile saline or $15 \mathrm{mM}$ kainate (Tocris, Bristol, UK) was stereotaxically injected into the left dorsal dentate gyrus (DG) at coordinates relative to bregma: anterioposterior $(\mathrm{AP})=-2.0 \mathrm{~mm}$, mediolateral $(\mathrm{ML})=-1.5 \mathrm{~mm}$, and relative to the cortical surface: dorsoventral $(\mathrm{DV})=-1.5 \mathrm{~mm}$. Following kainate injection, the occurrence of behavioral status epilepticus was verified by observation of mild convulsion, chewing, immobility, or rotations, as described before (Riban et al., 2002; Tulke et al., 2019). Ten mice died as a consequence of status epilepticus and further two were excluded due extreme hippocampal atrophy (Table 1). 
150 For optogenetic inhibition of CA1 principal cells, an AAV carrying the PACK silencer

151 with a red fluorescent marker mCherry under the control of the $\mathrm{Ca}^{2+} /$ calmodulin-

152 dependent kinase II alpha (CaMKIla) promoter (AAV9.CamKIla:SthK-P2A-bPAC-

153 mCherry referred to as AAV9.CaMKIla:PACK-mCherry) was injected into the right

154 hippocampus. In order to attribute the effects of AAV9.CaMKIla:PACK-mCherry to its

155 components, we included three control groups, in which mice were injected with (1) a

156 construct carrying the adenylyl cyclase bPAC but lacking the SthK channel

157 (AAV9.CamKIla:bPAC-mCherry), (2) a construct carrying only mCherry

158 (AAV9.CamKIla:mCherry), or (3) no virus (for sample sizes, see Table 1). The viral

159 constructs were injected into the right dorsal $C A 1$ area $(A P=-2.0 \mathrm{~mm}, \mathrm{ML}=-1.3 \mathrm{~mm}$,

$160 \mathrm{DV}=-1.15 \mathrm{~mm}$ ). The volume of the injected virus was adjusted to achieve an optimal

161 and comparable expression pattern in CA1: (1) PACK - $300 \mathrm{~nL}$, (2) bPAC - 250-300

$162 \mathrm{~nL},(3) \mathrm{mCherry}-300 \mathrm{~nL}$ with 1:5 dilution. All viral constructs were obtained from the

163 Viral Core Facility, Charité - Universitätsmedizin Berlin, Germany.

\section{Electrode and optic fiber implantations}

165 Implantations were performed 14-19 days after intrahippocampal injections as

166 described previously by Janz et al. (2018). For local field potential (LFP) analysis, we

167 implanted a Teflon-coated platinum-iridium wire electrode (125 $\mu \mathrm{m}$ diameter; World

168 Precision Instruments, Sarasota, Florida, USA) into each hippocampus: AAV-injected

169 right $\mathrm{CA} 1(\mathrm{AP}=-2.0 \mathrm{~mm}, \mathrm{ML}=-1.4 \mathrm{~mm}, \mathrm{DV}=-1.1 \mathrm{~mm})$ and saline/kainate-injected

170 left $\mathrm{DG}(\mathrm{AP}=-2.0 \mathrm{~mm}, \mathrm{ML}=+1.4 \mathrm{~mm}$, $\mathrm{DV}=-1.6 \mathrm{~mm})$. An optic fiber (ferrule 1.25

$171 \mathrm{~mm}$, cannula $200 \mu \mathrm{m}$ diameters; Thorlabs Inc., Newton, New Jersey, USA) was

172 implanted into the AAV-injected CA1, adjacent to the electrode at a $30^{\circ}$ angle $(\mathrm{AP}=$

$173-2.0 \mathrm{~mm}, \mathrm{ML}=-2.4 \mathrm{~mm}, \mathrm{DV}=-1.0 \mathrm{~mm}$ ). Two stainless steel screws (DIN 84;

174 Schrauben-Jäger, Landsberg, Germany) were implanted above the frontal cortex to 
175 provide a reference and ground. Electrodes and screws were soldered to a micro-

176 connector (BLR1-type) and fixed with dental cement (Paladur, Kulzer GmbH, Hanau,

177 Germany). The electrode and optic fiber positions were confirmed by post hoc histology

178 (Fig.1-1). Five mice were excluded from LFP analysis due to electrode/optic fiber

179 locations in the cortex above CA1. Three mice died following implantation procedures

180 (Table 1).

181 Electrophysiological recordings and optogenetic manipulations

182 Three-hour-long LFPs were acquired from freely moving mice in the period of 19-40

183 days after intrahippocampal injections. For LFP recordings, mice were connected to a

184 miniature preamplifier (MPA8i, Smart Ephys/ Multi Channel Systems, Reutlingen,

185 Germany). Signals were amplified 1000-fold, bandpass-filtered from $1 \mathrm{~Hz}$ to $5 \mathrm{kHz}$ and

186 digitized with a sampling rate of $10 \mathrm{kHz}$ (Power1401 analog-to-digital converter, Spike2

187 software, Cambridge Electronic Design, Cambridge, UK).

188 For each AAV9-injected mouse (with PACK, bPAC or mCherry), we acquired reference

189 LFPs before, in between, and after illumination experiments. LFPs with one-hour

190 illumination at $0.05 \mathrm{~Hz}$ or $0.1 \mathrm{~Hz}$ were recorded twice per frequency on separate days.

191 Each mouse represents a biological replicate ( $n=3-7$ per group, Table 1$)$ and the

192 number of recordings per mouse a technical replicate ( $n=2$ per recording type). The

193 sessions with illumination comprised one-hour pre-recording, 'light ON' phase during

194 the second hour and post-recording during the third hour. During the 'light ON' phase,

195 blue light pulses $\left(460 \mathrm{~nm}, \sim 80 \mathrm{~mW} / \mathrm{mm}^{2}, 10 \mathrm{~ms}\right.$ pulse duration, blue LED from

196 Prizmatix Ltd. Givat-Shmuel, Israel) were applied every 10 or 20 seconds. To hinder

197 rebound excitation resulting from the illumination off-set (Mahn et al., 2016; Bernal

198 Sierra et al., 2018), each light pulse had a $5 \mathrm{~ms}$ ramp-like termination (within pulse

199 fade-off). Furthermore, during the last 10 minutes of the 'light ON' phase, 5 ms light 
200 pulses were applied with gradually reducing intensity (within recording fade-off). The 201 light pulse duration and frequencies were selected based on previous work by Bernal 202 Sierra et al. (2018), who demonstrated that shining $5 \mathrm{~ms}$ light pulses with $0.05 \mathrm{~Hz}$ in 203 hippocampal slices provided long-lasting stable inhibition of current-elicited spiking in 204 PACK-expressing CA1 pyramidal cells.

Kainate-injected mice, which were implanted with intrahippocampal electrodes but did not receive a viral vector or an optic fiber, served as epileptic 'no virus' controls. Three207 hour recordings from these mice were performed on day 35 or 36 after kainate 208 injection.

\section{Analysis of local field potentials}

210 LFP data were visually inspected with Spike2 software and analyzed in detail using 211 Python 2.7. The line length, a sum of distances between successive data points, was 212 selected as a measure of the LFP waveform dimensionality since it is sensitive to 213 variations in both amplitude and frequency (Esteller et al., 2004). We calculated the 214 line length of downsampled data $(500 \mathrm{~Hz})$ by using the following equation, where $L$ is 215 the line length $(\mathrm{mV} / \mathrm{s}), x$ is the data-trace, $k$ is one data point, and $t$ is the recording 216 duration in seconds:

$$
L=\frac{\sum_{k=1}^{N} a b s[x(k-1)-x(k)]}{t}
$$

218 The line lengths were calculated for the first, second, and third hour of each recording.

219 In recordings with illumination, the line length calculations and spectral analysis were 220 done on LFP data recorded during the first 50 minutes; the last 10 minutes were 221 omitted due to light intensity fade-off. Furthermore, in recordings with illumination, the 222 line lengths shortly after the light pulses were compared to the line lengths directly before the light pulses. For this, two-second snippets were extracted before and after 
224 each light pulse during the first 50 minutes of the 'light ON' phase. In addition, two-

225 second snippets at corresponding time points were extracted in pre-recordings.

226 Subsequently, the mean "pre", "before pulse", and "after pulse" line lengths were

227 calculated for each recording session (2 per animal). Periods with electrographic

228 generalized seizures (GS), where hypersynchronous neuronal activity was

229 propagating across hemispheres followed by postictal depression, were removed from

230 analysis due to strongly altered LFP characteristics.

231 The frequency compositions of the LFPs are presented with power spectral densities

232 (PSD) and spectrograms generated by fast Fourier transform (FFT) of LFP raw data

233 (sampling rate $10 \mathrm{kHz}$ ). The PSDs were estimated by applying Welch's method in

234 Python 2.7 (scipy.signal.welch function), based on time averaging over

235 short periodograms (periodogram length $=10 x$ sampling rate). The oscillatory power

236 was calculated as the area under the PSD plot for the respective frequency ranges:

237 delta $(1-4 \mathrm{~Hz})$, theta $(4-12 \mathrm{~Hz})$, beta $(12-30 \mathrm{~Hz})$, gamma (30-120 Hz). For time-

238 frequency representation of the LFP power in a spectrogram, the Hanning window was

239 applied and data in the time-domain (length of FFT= 10x sampling rate) was broken

240 up into overlapping (overlap $=0.25 \mathrm{x}$ sampling rate) segments (segment length $=1 \mathrm{x}$

241 sampling rate).

\section{Analysis of epileptiform activity}

243 Downsampled hippocampal LFPs recorded from epileptic animals were analyzed in

244 detail using a custom-made semi-automated algorithm that detects and classifies

245 epileptiform activity (Heining et al., 2019). In the ihpKA mouse model, epileptiform

246 activity occurs as single sharp wave epileptiform spikes and as bursts, which are

247 clusters of many spikes (Riban et al., 2002). The algorithm classifies the bursts

248 according to their spike-load into low-load, medium-load, and high-load bursts as 
described by Heining et al. (2019). To assess the effect of PACK-mediated inhibition on seizure activity, we calculated the 'burst ratio', which is the duration of high-load bursts per total recoding time. The automatic detection of high-load bursts was verified by visual inspection of the LFP recordings. Sessions with GS were removed from the analysis due to long-lasting suppression of neuronal activity after such a seizure.

\section{Perfusion and tissue preparation}

After the last LFP recording (at day 30-40), the mice were deeply anesthetized and transcardially perfused with $0.9 \%$ saline followed by $4 \%$ paraformaldehyde in $0.1 \mathrm{M}$ phosphate buffer (PB, pH 7.4). The brains were dissected and post-fixated overnight in $4 \%$ paraformaldehyde, followed by sectioning (coronal plane, $50 \mu \mathrm{m}$ ) with a vibratome (VT100S, Leica Biosystems, Wetzlar, Germany). The slices were collected and stored in PB for immunohistochemistry.

\section{Immunohistochemistry}

262 To determine optic fiber and electrode positions, to visualize the hippocampal anatomy 263 after AAV9-injections, and to validate hippocampal sclerosis in ihpKA mice, we 264 performed immunohistochemistry with markers for neurons (NeuN) and astrocytes 265 (glial fibrillary acidic protein - GFAP). For the immunofluorescence staining, free266 floating sections were pre-treated with $0.25 \%$ TritonX-100 and $10 \%$ normal horse or 267 goat serum (Vectorlabs, Burlingame, California, USA) diluted in PB for $1 \mathrm{~h}$. 268 Subsequently, slices were incubated either with guinea-pig anti-NeuN (1:500; Synaptic 269 Systems, Göttingen, Germany) or rabbit anti-GFAP (1:500, Dako, Glostrup, Denmark) 270 overnight at $4^{\circ} \mathrm{C}$. Sections were rinsed and then incubated for $2.5 \mathrm{~h}$ in donkey anti271 guinea-pig or donkey anti-rabbit Cy5-conjugated secondary antibody (1:200, Jackson 272 ImmunoResearch Laboratories Inc., West Grove, Pennsylvania, USA), followed by 
273 extensive rinsing in PB. The sections were mounted on glass slides and coverslipped

274 with Immu-Mount ${ }^{T M}$ mounting medium (Thermo Shandon Ltd, Runcorn, UK).

275

\section{Image acquisition and histological analysis}

Tiled fluorescent images of the brain sections were taken with an Axiolmager 2 microscope (Carl Zeiss Microscopy GmbH, Jena, Germany) using a Plan- Apochromat 10x objective with numerical aperture 0.45 (Zeiss, Göttingen, Germany). The exposure times (Cy5-labeled NeuN: 5 s, Cy5-labeled GFAP: 3 s, mCherry: 300 ms) were kept constant for each staining to allow for comparisons across animals.

To assess the effect of AAV9-mediated delivery of PACK, bPAC and/or mCherry and long-term expression of these proteins on hippocampal histology, we measured the relative expression intensities of GFAP labeling in the mCherry-expressing dorsal CA1 at three positions along the anteroposterior axis $(-1.70 \mathrm{~mm},-1.94 \mathrm{~mm}$, and $-2.18 \mathrm{~mm}$ from bregma). The quantification was performed in Fiji Image J by drawing a polygonshaped area of interest (ROI) around mCherry expression in CA1 (str. oriens to str. radiatum) and taking the mean grey area of both mCherry and the GFAP labeling within this ROI. Areas with glial scarring around the implantations were excluded by adjusting the ROI. The same measurement was done in the contralateral CA1 by drawing a similar ROI, which avoided implant-related scars. For normalization, in each slice the local background was measured in a small square $\left(41457 \mu \mathrm{m}^{2}\right)$ in the cortex and subtracted from the mean grey areas of each ROI in CA1. Finally, mean expression intensities of mCherry and GFAP in left (saline-injected) and right (virus-injected) CA1 were presented for each animal. Furthermore, we quantified the width of the pyramidal cell layer in dorsal medial CA1 by measuring three perpendicular lines in the left and in the right CA1 of each NeuN-labeled section (3 sections per mouse, AP: -1.70 mm, - 
$2971.94 \mathrm{~mm},-2.18 \mathrm{~mm}$ from bregma) and compared the mean width of the left and right

298 CA1 pyramidal cell layer.

299 In ihpKA mice, the presence of hippocampal sclerosis in the kainate-injected

300 hippocampus was confirmed in NeuN-labeled sections showing granule cell dispersion

301 and cell loss in CA1 and CA3 and in GFAP-labeled sections demonstrating astrogliosis.

\section{Statistical analysis}

303 Data were tested for statistical significance with GraphPad Prism 8 software

304 (GraphPad Software Inc.). To determine how the line length and spectral power 305 changed during the second and third hour of LFP recordings, the baseline value (in the 306 first hour) was subtracted from the original values and tested for significance using a 307 one-sample t-test with a Bonferroni correction of the significance level $(\alpha=0.025$ for two 308 comparisons). A paired t-test was used for comparing two matched groups of 309 parametric data. Comparisons of more than two parametric data sets were performed 310 either with a one-way ANOVA or with repeated-measures (RM) ANOVA, in case of 311 matched groups. If an ANOVA indicated that not all group means were equal, Tukey's 312 multiple comparisons test was performed additionally. Friedman's test (matched) or 313 Kruskal-Wallis test (non-matched) with Dunn's post hoc were applied for comparing 314 three groups of non-parametric data. Pearson's correlation coefficient was used to 315 measure the strength of association between two variables. Significance thresholds 316 were set to: ${ }^{*} p<0.05,{ }^{* *} p<0.01$ and ${ }^{* * *} p<0.001$. For all parametric data, mean and SEM 317 are given; for non-parametric data, median with interquartile range (IQR) are reported. 


\section{Results}

\section{Light-activated PACK reduces the activity of pyramidal cells in vivo}

To verify the inhibitory action of the PACK silencer in awake mice, we targeted

321 hippocampal principal cells by locally injecting AAV9.CaMKIla.PACK-mCherry into the

322 CA1 area of the dorsal hippocampus (Fig. 1A) and enabled illumination onto these neurons via an implanted optic fiber (Fig. 1B). To test whether applying short light pulses $(10 \mathrm{~ms})$ at low frequencies in vivo results in sustained inhibition of PACKexpressing CA1 neurons as previously demonstrated in vitro (Bernal Sierra et al., 2018), we shined blue light at $0.05 \mathrm{~Hz}$ and $0.1 \mathrm{~Hz}$ for one hour. The light ON phase was enclosed by a pre- and post-recording, one hour each (Fig. 1C). Following the recording phase, histological analysis revealed that expression of PACK-mCherry was restricted to pyramidal neurons in CA1 with labeling in cell bodies and dendrites

330 (Fig.1D). For LFP analysis, we only included mice, which had the optic fiber and 331 recording electrode positioned in CA1 (Supplementary Fig. 1, n=6).

332 First, we recorded three-hour reference LFPs in each mouse (Fig. 1E) to control for 333 the change in LFP characteristics occurring without any manipulation due to 334 habituation - reduction in arousal and exploratory behavior (Cabib et al., 1990; Hinman 335 et al., 2011). Quantification of the LFP signal by determination of the line length, a 336 measure for LFP magnitude (see Materials and Methods), revealed an innate decrease 337 during the reference recordings (first hour: set to zero; second hour: $-7.98 \pm 1.01 \mathrm{mV} / \mathrm{s}$, 338 one-sample t-test: $t=7.93, n=12$ trials, $p<0.0001, \alpha=0.025$; third hour: $-10.54 \pm 4.35$ $339 \mathrm{mV} / \mathrm{s}$, one-sample t-test: $\mathrm{t}=8.38, \mathrm{n}=12$ trials, $\mathrm{p}<0.0001, \alpha=0.025$; Fig. 1F). Next, we 340 activated PACK with intermittent light application. During $0.05 \mathrm{~Hz}$ illumination, LFP 341 magnitude was reduced directly after light pulses, followed by periods of recovery (Fig. 342 1G). The mean line length significantly decreased during $0.05 \mathrm{~Hz}$ illumination (50 
343 minutes $)$ compared to the pre-recording $(-10.33 \pm 2.18 \mathrm{mV} / \mathrm{s}$, one-sample t-test, $\mathrm{t}=4.73$,

$344 \mathrm{n}=12$ trials, $\mathrm{p}=0.0006, \alpha=0.025$; Fig. $1 \mathrm{H}$ ). Illumination with $0.1 \mathrm{~Hz}$ provided a stable

345 reduction of the LFP magnitude (Fig. 1G) with a strong decrease in the line length

346 during the light $\mathrm{ON}$ phase $(-19.42 \pm 3.14 \mathrm{mV} / \mathrm{s}$, one-sample $\mathrm{t}$-test: $\mathrm{t}=6.18, \mathrm{n}=12$ trials,

$347 p<0.0001, \alpha=0.025 ;$ Fig. 1J). The change of line length from the first to the second hour

348 was significantly different in the three recording types (reference: $-7.98 \pm 1.01 \mathrm{mV} / \mathrm{s}$,

$3490.05 \mathrm{~Hz}:-10.33 \pm 2.18 \mathrm{mV} / \mathrm{s}, 0.1 \mathrm{~Hz}:-19.42 \pm 3.14 \mathrm{mV} / \mathrm{s}, \mathrm{RM}$ ANOVA: F=14.40, n=12,

$350 p=0.012$ ). The drop of line length was notably higher in the $0.1 \mathrm{~Hz}$ recordings than in

351 the respective reference recordings (Tukey's multiple comparison test: $p=0.003$ ),

352 whereas in the sessions with $0.05 \mathrm{~Hz}$ it was similar to the reference recordings (Tukey's

353 multiple comparison test: $p=0.33)$.

354 To test the reliability of the PACK-mediated inhibition, we further analyzed the 355 responses to light pulses applied at $0.05 \mathrm{~Hz}$ and $0.1 \mathrm{~Hz}$. We extracted two-second LFP 356 snippets before and after each light pulse (Fig. 2A), plotted their overlay (Fig. 2B, D), 357 and calculated the mean of the "before pulse" and "after pulse" line lengths for each 358 recording session (Fig. 2C, E). We also extracted two-second LFP snippets at 359 corresponding time points from respective pre-recordings ("pre") to serve as a 360 baseline. In the $0.05 \mathrm{~Hz}$ session, reduction of LFP magnitude was reliable and 361 reversible since there was a reduction after each light pulse, followed by a complete 362 recovery during the 20 -second interval between subsequent pulses (Fig. 2B). The 363 mean "after pulse" line length was significantly smaller than "pre" and "before pulse" 364 line length in $0.05 \mathrm{~Hz}$ sessions ("pre": $48.67 \pm 3.83 \mathrm{mV} / \mathrm{s}$, "before pulse": $48.38 \pm 4.02$ $365 \mathrm{mV} / \mathrm{s}$, "after pulse": $32.39 \pm 2.31 \mathrm{mV} / \mathrm{s}$, RM ANOVA: $\mathrm{F}=17.83, \mathrm{n}=12, \mathrm{p}=0.0001$, Tukey's 366 multiple comparisons test: $p<0.001$; Fig. 2C). Shining $10 \mathrm{~ms}$ light pulses every 10 367 seconds provided a persistent reduction in LFP amplitude (Fig. 2D). In the $0.1 \mathrm{~Hz}$ 
sessions, the line length was lower during the whole light ON period, including "after pulse" and "before pulse" snippets, suggesting a constant inhibitory effect ("pre”: 49.99 $\pm 4.80 \mathrm{mV} / \mathrm{s}$, "before pulse": $35.48 \pm 3.13 \mathrm{mV} / \mathrm{s}$, "after pulse": $28.69 \pm 2.23 \mathrm{mV} / \mathrm{s}, \mathrm{RM}$ ANOVA: $F=18.02, n=12, p=0.0019$, Tukey's multiple comparisons test: "pre" vs. "before pulse" $p=0.0259$, "pre" vs. "after pulse" $p=0.003$; Fig. 2E). In summary, shining blue light at $0.1 \mathrm{~Hz}$ onto PACK-expressing CA1 neurons in vivo resulted in a sustained reduction of the net neuronal activity throughout the light ON period.

Next, we examined how light-induced PACK-activation in principal cells alters oscillatory activity in CA1. The most dominant oscillations measured in the hippocampus of freely behaving rodents are theta $(4-10 \mathrm{~Hz})$ and gamma $(30-120 \mathrm{~Hz})$ waves (Bragin et al., 1995; Buzsáki et al., 2003; Colgin, 2016). The theta and gamma peaks are prominent in spectrogram snippets (Fig. 3A) and in the mean PSD plot (PSD averaged across recordings; Fig. 3B) of the reference recordings acquired from PACK mice. We quantified the oscillatory power by taking the area under the curve of the PSD plot in the respective frequency range. The power of theta, beta, and gamma oscillations decreased significantly from the first to second hour presumably due to habituation (theta: $-0.12 \pm 0.02$, beta: $-0.13 \pm 0.02$, gamma: $-0.16 \pm 0.03$, one-sample t-test, $n=12, p:<0.0001-0.003, \alpha=0.006 ;$ Fig. 3E)

Applying light pulses at $0.1 \mathrm{~Hz}$, transiently reduced the spectral power of frequencies above $\sim 10 \mathrm{~Hz}$ (Fig. 3C). The mean PSD during the 50-minute $0.1 \mathrm{~Hz}$ light ON phase was visibly reduced compared to pre- and post-recordings, especially in the beta and gamma ranges (Fig. 3D). Quantification of the power change revealed a significant drop of theta, beta, and gamma power during $0.1 \mathrm{~Hz}$ illumination (theta: $-0.23 \pm 0.06$, beta: $-0.35 \pm 0.07$, gamma: $-0.46 \pm 0.07$, one-sample t-test, $p<0.0001-0.0038, \alpha=0.006$, $\mathrm{n}=12$ trials; Fig. 3F). The reduction of beta and gamma power during $0.1 \mathrm{~Hz}$ illumination 
was significantly stronger than the inherent decline in respective reference recordings (beta: paired t-test, $t=3.63, n=12$ trials, $p=0.004$, gamma: paired t-test, $t=4.47, p=0.001$, $\alpha=0.0125 ;$ Fig. 3G). These data indicate that PACK-mediated inhibition of CA1 neurons in vivo alters the power of beta and gamma oscillations.

\section{Light-dependent hyperactivity in bPAC-expressing mice}

Activation of the PACK silencer includes cAMP production by soluble bPAC, which then opens the co-expressed SthK potassium channels in the cell membrane. The second messenger molecule, cAMP, is an important component of intracellular signaling, regulating the plasticity and excitability of neurons (Huang et al., 1994; Weisskopf et al., 1994; Gruart et al., 2012; Fukaya et al., 2021). Therefore, it is crucial to investigate whether activation of bPAC alone affects network excitability.

To this end, we targeted bPAC with the AAV9.CaMKII viral vector to CA1 neurons and repeated the experiments like with PACK mice (Fig. 4A-D). Surprisingly, light-activation of bPAC at $0.1 \mathrm{~Hz}$ led to sustained neuronal hyperactivity that is clearly visible in LFP snippets (Fig. 4E) as well as in corresponding spectrograms (Fig. 4G). The line length was significantly increased during $0.1 \mathrm{~Hz}$ illumination $(12.10 \pm 3.55$, one-sample t-test: $\mathrm{t}=3.41, \mathrm{n}=12, \mathrm{p}=0.0058, \alpha=0.025 ; \mathrm{Fig} .4 \mathrm{~F})$. The oscillations, which were mainly altered by bPAC activation were beta and gamma with significantly elevated power during illumination (beta: $0.50 \pm 0.17$, one-sample t-test: $t=3.03, n=12, p=0.011$, gamma: 0.34 \pm 0.096 , one-sample t-test: $t=3.52, n=12, p=0.0048, \alpha=0.0125 ;$ Fig. $4 \mathrm{H}-\mathrm{I})$. The theta oscillatory power increased slightly but not significantly during light-induced bPAC activation $(0.25 \pm 0.13$, one-sample t-test: $t=1.91, n=12, p=0.08, \alpha=0.0125 ;$ Fig. $4 \mathrm{I})$. In the post-recording, LFP magnitude and gamma power dropped again, suggesting that the neuronal hyperactivity was reversible and related to light-induced elevation of cAMP levels (line length: $-8.86 \pm 2.80 \mathrm{mV} / \mathrm{s}$, one-way $\mathrm{t}$-test, $\mathrm{t}=3.16, \mathrm{n}=12, \mathrm{p}=0.0091$, 
$418 \alpha=0.025$; Fig. 4F; gamma power: $-0.11 \pm 0.04 \mathrm{mV} / \mathrm{s}$, one-way t-test, $\mathrm{t}=2.68, \mathrm{n}=12, \mathrm{p}=$

0.02; Fig. 4I). The elevation in neuronal activity was not induced by blue light per se since mice injected with AAV9.CaMKII.mCherry did not show any changes in the LFP

421 signal during illumination (Supplementary Fig. 2). In summary, light-induced activation 422 of bPAC without the SthK channel co-expression transiently elevates neuronal activity 423 in CA1.

424 Spontaneous generalized seizures arising in PACK- and bPACexpressing mice

426 In healthy control mice, which received intrahippocampal saline and recording 427 electrodes, epileptiform activity is normally absent in the LFP (Twele et al., 2017). 428 Unexpectedly, in the majority of the PACK (5 out of 6 ) and all of the bPAC mice $(n=6)$, 429 hypersynchronous activity, spreading across both hemispheres, arose at least once 430 during LFP recordings (Fig. 5A, B, E). Most of these electrographic generalized 431 seizures were accompanied by behavioral correlates such as freezing, nodding, 432 forelimb clonus, or rearing according to the Racine scale (Racine, 1972).

433 The occurrence of generalized seizures in baseline recordings ("ref" and "pre") 434 indicates seizure initiation independent from light-induced PACK (Fig. 5C) and bPAC 435 (Fig. 5D) activation. Illumination of bPAC-expressing neurons increased the median of 436 average seizure count in three hours compared to post-recordings ("pre": 0.09 [0, 0.48], 437 "light ON": 1.13 [0.45, 3.94], "post": 0 [0, 0.86], Friedman test: Fr= 6.38, n=6, p=0.041, 438 Dunn's post hoc: $p=0.042$; Fig. 5D). The median number of generalized seizures in all 439 recordings was the highest in bPAC mice (3.50 [1.00, 9.75]; Fig. 5E). Most PACK mice 440 experienced one generalized seizure throughout all the recordings (in total 9-28h), $441(1.00[0.75,2.75], n=6)$, whereas mCherry $(n=4)$ and 'no virus' $(n=7)$ control mice had 
442 no generalized seizures (Fig. 5E). These results suggest that the dark activity of bPAC

443 is responsible for spontaneous generalized seizures arising in mice that express PACK

444 or bPAC in CA1 pyramidal cells.

\section{Histological abnormalities in PACK- and bPAC-expressing CA1}

An optogenetic tool suitable for long-term in vivo experiments should preserve the normal physiology and histology in the target area. For histological analysis, PACK, bPAC, and mCherry mice were perfused after the last LFP recording, 30-35 days after the intrahippocampal virus and saline injections. Coronal sections were immunolabeled with anti-NeuN and anti-GFAP to investigate the histology of neurons and astrocytes respectively.

To our surprise, we found notable widening of the pyramidal cell layer in PACKexpressing CA1 (Fig. 6A). The mean width of the pyramidal cell layer in PACKexpressing right CA1 was significantly higher than in the saline-injected left side (right CA1: $71.66 \pm 2.01 \mu \mathrm{m}$; left CA1: $60.68 \pm 0.54 \mu \mathrm{m}$, paired t-test: $t=5.25, n=8, p=0.0012$, Fig. 6B). The pyramidal cell layer was also significantly wider in bPAC-expressing CA1 compared to its contralateral counterpart (right CA1: $84.73 \pm 2.23 \mu \mathrm{m}$, left CA1: 65.27 $\pm 0.94 \mu \mathrm{m}$, paired t-test: $\mathrm{t}=8.42, \mathrm{n}=6, \mathrm{p}=0.0004$; Fig. $6 \mathrm{C})$. Mice, which received the same viral vector, carrying just the reporter mCherry, had similar pyramidal cell layer widths in the left and right hippocampus (right CA1: $70.96 \pm 3.53 \mu \mathrm{m}$, left CA1: 69.10 $\pm 3.17 \mu \mathrm{m}$, paired t-test: $\mathrm{t}=0.95, \mathrm{n}=4, \mathrm{p}=0.41$; Fig. $6 \mathrm{D}$ ). Cell dispersion, taken as the difference between right and left CA1 width, was the highest in bPAC-expressing CA1 $(19.45 \pm 2.31 \mu \mathrm{m})$, followed by PACK-expressing CA1 $(10.98 \pm 1.84 \mu \mathrm{m})$, and lacking in mCherry-expressing CA1 $(1.86 \pm 1.94 \mu \mathrm{m}$, one-way ANOVA: $F(3,18)=12.51$, $p=0.0006$, Tukey's multiple comparisons test: PACK vs. bPAC $p=0.0302, \quad$ PACK vs. mCherry $p=0.0397$, bPAC vs. mCherry $p=0.0005$; Fig. $6 E$ ). These findings led us to 
467 conclude that bPAC, and not the viral vector itself, is inducing the cell dispersion in the

468 CA1 pyramidal cell layer.

469 GFAP labeling in hippocampal sections shows salient chronic astrogliosis in the PACKexpressing CA1 area (Fig. 6F, F2). The comparison of GFAP labeling in left and right

471 hippocampi revealed strongly elevated GFAP intensity in the PACK-expressing CA1

472 (right CA1: $8.70 \pm 0.46$, left $C A 1: 3.62 \pm 0.41$, paired $t$-test: $t=13.54, n=5, p=0.0002$;

473 Fig. 6G). In bPAC mice, we also found notable astrogliosis in the right bPAC474 expressing hippocampus (right CA1: $11.62 \pm 1.03$, left CA1: $3.73 \pm 0.34$, paired t-test: $475 \mathrm{t}=6.01, \mathrm{n}=6, \mathrm{p}=0.0018$, Fig. $6 \mathrm{H})$. In mCherry mice, the GFAP intensity was slightly but 476 significantly elevated in the right hippocampus (right CA1: $3.98 \pm 0.61$, left CA1: $3.15 \pm$ 477 0.53, paired t-test: $t=4.12, n=4, p=0.026$; Fig. $6 \mathrm{I})$. Thus, it could be that either the viral 478 vector or the presence of an electrode and an optic fiber contributed to the glial scarring 479 in the right hippocampus. There was no significant correlation between the strength of 480 mCherry-expression and GFAP intensity in PACK, bPAC, and mCherry mice 481 (Pearson's correlation: $r=0.35, p=0.2$; Fig. 6J). However, the three groups formed 482 separate clusters with: (1) mCherry mice having medium mCherry expression but the 483 lowest GFAP intensity, (2) PACK mice having the lowest mCherry expression but 484 medium GFAP intensity, and (3) bPAC mice having the strongest mCherry expression 485 and the highest GFAP intensity. Taken together, it seems like bPAC expression is the 486 main factor inducing chronic astrogliosis in PACK and bPAC mice, while the viral vector 487 and hippocampal implantations might contribute additionally. 


\section{PACK/bPAC expression in the contralateral hippocampus prevents} seizure spread in chronically epileptic mice

490

491

To find out if the PACK silencer could be used to limit the spread of epileptiform activity, we targeted PACK to the CA1 principal cells, contralateral to inpKA treatment. The inpKA mouse model recapitulates the main pathological features of MTLE, i.e. spontaneous recurrent seizures associated with hippocampal sclerosis (Bouilleret et al., 1999). Most inpKA mice have epileptiform activity that occurs in form of bursts originating in the seizure focus and propagating into the contralateral hippocampus (Meier et al., 2007; Janz et al., 2018; Paschen et al., 2020). Unexpectedly, all our PACK-injected inpKA mice $(n=5)$ were free of contralateral seizures already in baseline recordings before light-activation of PACK.

We detected the epileptiform bursts with high spike load using an automated algorithm (Heining et al., 2019) and quantified the burst ratio, the fraction of time spent in bursts, during the respective recording (Fig. 7). Although the epileptiform bursts occurred frequently in the ipsilateral hippocampus of PACK ihpKA mice (mean burst ratio in "pre": $0.19 \pm 0.02$, "0.1 Hz": $0.18 \pm 0.02$, "post": $0.20 \pm 0.02, \mathrm{n}=5)$, the contralateral hippocampus was devoid of seizure activity (Fig. 7E-G). Similarly, in bPAC inpKA mice, epileptiform bursts were detected in the ipsilateral (mean burst ratio in "pre": $0.11 \pm$ 0.03, "0.1 Hz": $0.07 \pm 0.03$, "post": $0.13 \pm 0.05, n=3$; Fig. $7 \mathrm{I})$ but not the contralateral hippocampus (Fig. 7J). Neither the light-induced activation of bPAC nor the whole PACK construct had any effect on the burst ratios in the ipsilateral or contralateral hippocampus (Fig. 7E-J).

We compared the burst ratios in PACK and bPAC mice to 'no virus' mice $(n=4)$ in a three-hour recording 33-36 days after kainate to clarify whether the lack of contralateral 
512 seizures affects the seizure burden in the kainate-injected ipsilateral hippocampus

513 (Fig. 7K-M). There was no significant difference in the ipsilateral burst ratios (PACK:

$5140.19 \pm 0.03$, bPAC: $0.11 \pm 0.05$, no virus: $0.13 \pm 0.03$, one-way ANOVA: $F=1.53, n=3-$

515 5, p=0.26; Fig. 7L), whereas the mean contralateral burst ratio was evidently higher in 516 'no virus' mice than in PACK and bPAC mice (PACK: $0.00 \pm 0.00$, bPAC: $0.00 \pm 0.00$, 517 no virus: $0.12 \pm 0.04$, one-way ANOVA: $F=6.26, n=3-5, p=0.017$, Dunnett's multiple 518 comparisons test: PACK vs. no virus $p=0.018$, bPAC vs. no virus $p=0.036$; Fig. $7 M$ ). 519 These results suggest that the dark activity of bPAC in CA1 pyramidal neurons 520 prevents the spread of epileptiform activity to the bPAC-expressing areas. The activity 521 of CA1 pyramidal cells is thus critical for seizure propagation into the contralateral 522 hippocampus. Additionally, the absence of contralateral bursts does not affect the 523 seizure burden in the sclerotic hippocampus.

\section{Discussion}

525 The present study provides a detailed characterization of a novel two-component potassium channel-based silencer, PACK, and its long-term application in freely 527 moving mice. We targeted the PACK construct to hippocampal CA1 with an 528 AAV9.CaMKII vector, resulting in robust expression of PACK-mCherry in principal neurons of this area. We report that PACK is a suitable silencer to reduce the activity of hippocampal neurons in vivo by applying short blue light pulses via an implanted optic fiber. Previously, Bernal Sierra et al. (2018) demonstrated in acute hippocampal

532 slices that shining $5 \mathrm{~ms}$ blue light pulses at $0.05 \mathrm{~Hz}$ onto PACK-expressing CA1 cells 533 was sufficient to abolish spiking elicited by current injections. In our freely moving mice, 534 applying $10 \mathrm{~ms}$ light pulses at $0.05 \mathrm{~Hz}$ provided an unstable lowering of the LFP magnitude with periods of recovery. However, with an increased illumination frequency 
537 phase. This is a significant improvement compared to microbial chloride and proton

538 pumps, which require continuous illumination and already exhibit declining

539 photocurrent amplitudes within one minute (Mattis et al., 2012; Wiegert et al., 2017).

540 Regarding oscillatory activity in CA1, light-induced PACK activation in CA1 pyramidal

541 cells mainly reduced the power of gamma and beta oscillations. Hippocampal gamma

542 rhythm depends on the firing of pyramidal cells and their synchronized dendritic and

543 perisomatic inhibitory input, which originate from local somatostatin- and parvalbumin-

544 positive interneurons, respectively (Tukker et al., 2007; Antonoudiou et al., 2020).

545 Constant reduction of pyramidal cell activity consequently decreases local interneuron

546 activity (Gridchyn et al., 2020), which would explain the lower power of gamma

547 oscillations during PACK-mediated inhibition. The beta power was probably reduced

548 for the same reason, as the activity of local CA1 interneurons is also crucial for beta

549 oscillations (Rangel et al., 2016). PACK-mediated inhibition affected theta oscillations

550 to a much lesser extent, probably while theta rhythm is predominantly driven by

551 GABAergic and cholinergic inputs from the medial septum while depending less on

552 local excitation and inhibition (Hangya et al., 2009; Fuhrmann et al., 2015; Müller and

553 Remy, 2018).

554 Using soluble bPAC as the light-sensitive domain in the PACK tool is favorable to 555 ensure high light sensitivity (Beck et al., 2018; Bernal Sierra et al., 2018). However, 556 this advantage seems to have come with dark activity, which is probably the reason for 557 the side effects we observed in PACK- and bPAC-expressing hippocampi in vivo. The 558 harmful effects of chronic bPAC-expression in the hippocampal CA1 area included 559 pyramidal cell layer widening, chronic astrogliosis, and spontaneous generalized 560 seizures. Mice that received the AAV9 vector carrying only mCherry under the CaMKII 561 promoter did not show these side effects, except for astrogliosis, which could be due 
562 to implantation-related scarring. Based on these findings, we conclude that the viral 563 vector and the fluorescent marker alone were not detrimental. As bPAC mice tended 564 to have higher expression of bPAC-mCherry, more prominent CA1 dispersion, stronger astrogliosis, and higher occurrence of generalized seizures than PACK mice, chronic cAMP elevation may be the underlying cause of these adverse effects.

The second messenger molecule cAMP has several molecular targets in neurons,

568 including protein kinase A (PKA), exchange protein activated by cAMP (Epac), and cAMP-gated ion channels. Through these pathways, cAMP regulates fundamental physiological processes such as growth, metabolism, migration, apoptosis, gene transcription, neurotransmission, and plasticity (for review, see Nguyen \& Woo, 2003; Cheng et al., 2008; Antoni, 2012). Altered gene expression by activation of cAMP-

573 responsive element (CRE) could explain histological changes and seizure activity in 574 bPAC mice as the CRE-transcriptional pathway is involved in acute and chronic phases 575 of epilepsy (Huang et al., 1994; Hansen et al., 2014; Choi et al., 2016; Conte et al., 2020). The nuclear distribution element-like 1 (NDEL1) protein, transcribed in an 577 activity-dependent manner by the cAMP response element-binding (CREB) protein, 578 has been hypothesized to contribute to pathophysiological alterations of MTLE. NDEL1 579 is robustly and persistently elevated in the mouse hippocampus, including CA1 580 neurons, after pilocarpine-induced status epilepticus (Choi et al., 2016; Zhu et al., 581 2020). A conditional knockout of the NDEL1 gene in CA1 of adolescent mice leads to 582 dispersion and increased intrinsic excitability of CA1 pyramidal cells (Jiang et al., 583 2016), resembling what we observed in our bPAC mice.

584 Light-activation of bPAC in vivo strongly increased LFP magnitude and the oscillatory 585 power of beta and gamma in the CA1 region. The neuronal activity dropped 586 immediately after the illumination phase, suggesting a temporary depolarizing effect 
587 via a cyclic nucleotide-gated channel or induction of short-term potentiation. Local cAMP elevation induces presynaptic potentiation by promoting the accumulation of calcium channels close to release sites, thus increasing the release probability

590 (Midorikawa and Sakaba, 2017; Vaden et al., 2019; Fukaya et al., 2021). Presynaptic 591 elevation of cAMP via light-activation of synapse-targeted bPAC (synaptoPAC) was 592 sufficient to trigger potentiation at the mossy fiber-CA3 synapse but not in the CA3593 CA1 synapse (Oldani et al., 2021). Although cAMP-mediated presynaptic potentiation 594 is not exhibited by all hippocampal synapses, it is present in the CA1-subicular bursting neuron synapse (Wozny et al., 2008). Therefore, temporarily increased transmission at the CA1-subiculum synapses might have contributed to light-induced hyperactivity 597 in bPAC-expressing CA1 in vivo. Alternatively, cAMP binding on hyperpolarization598 activated cyclic nucleotide-gated (HCN) cation channels might have increased pyramidal cell excitability because (1) HCN channels are abundantly expressed in hippocampal neurons, and (2) the inward currents through HCN channels depolarize 601 the membrane (Santoro et al., 2000; Lörincz et al., 2002; He et al., 2014). Furthermore, $602 \mathrm{HCN}$ channels are thought to initiate rhythmic firing (Nolan et al., 2004; He et al., 2014), 603 which could theoretically explain elevated beta and gamma oscillations during light604 induced bPAC activation in vivo. Future experiments with probes or tetrodes could 605 potentially clarify the mechanism of bPAC-mediated excitation and PACK-mediated 606 inhibition at a single-unit level.

607 In the last part of our study, we applied the PACK silencer in chronically epileptic inpKA 608 mice, which usually exhibit spontaneous seizures in both hippocampi (Meier et al., 609 2007; Janz et al., 2018; Paschen et al., 2020). We targeted PACK to the contralateral 610 hippocampus (opposite to kainate injection) to determine if we can interfere with 611 seizure spread between the two hippocampi. To our surprise, there was no propagation 
612 of seizure activity from the kainate-injected hippocampus to the PACK-expressing 613 contralateral side, even before light-activation of PACK. We saw the same in bPAC 614 mice, which were also lacking contralateral seizures already in the baseline recordings.

615 Surprisingly, prolonged bPAC expression in saline-injected mice induced generalized 616 seizures, whereas it prevented seizure spread in kainate-injected mice. Hypothetically, 617 bPAC-dependent cAMP elevation and subsequent HCN channel activation could explain contradictory findings regarding epileptogenicity, since HCN channels have also been found pro- and anti-epileptic (Bender et al., 2003; Lewis and Chetkovich, 2011; Noam et al., 2011; Stegen et al., 2012). Future work comparing gene expression and hippocampal slice electrophysiology in saline- and kainate-treated mice with and

622 without bPAC-expression would be needed to address the mechanism of cAMP623 associated seizure induction and prevention.

624 Our results suggest that the expression of bPAC affects the physiology of hippocampal principal cells in the absence of blue light. Functional dark activity of soluble bPAC has

626 also been reported by others, who utilized a fluorescent PKA-sensor to detect 627 intracellular cAMP levels in bPAC-expressing CA1 cells in organotypic hippocampal 628 cultures (personal communication with O. Constantin, group of Prof. Oertner). For 629 reduced dark activity, the soluble bPAC from the Beggiatoa bacterium could be 630 replaced by another photoactivated adenylyl cyclase (PAC). There are several PACs 631 available from other microorganisms, which have lower light sensitivity but still provide 632 sufficient potassium current when coupled to SthK (Bernal Sierra et al., 2018). 633 Alternatively, a red-shifted bPAC with reduced dark activity could be used (Stierl et al., 634 2014). Another approach would be targeting a PAC to the cell membrane. For example, 635 membrane-bound guanylyl cyclase rhodopsins, which have mutated to be adenylyl 636 cyclases, virtually lack dark activity (Scheib et al., 2018; Henß et al., 2021). In these 
637 approaches, the possibility of SthK channel activation by intrinsic cAMP remains.

638 Accordingly, expression of SthK without bPAC in body wall muscle cells of C.elegans

639 was already sufficient to see a behavioral change resulting from muscle

640 hyperpolarization (Henß et al., 2021). This problem could be overcome by engineering 641 SthK variants with mutations in the cAMP binding site, resulting in a channel with 642 reduced cAMP affinity.

643 In conclusion, we showed in awake mice that the potassium channel-based 644 optogenetic silencer PACK reliably reduces hippocampal neuronal activity in a light645 dependent manner. In contrast to other optogenetic inhibitors, PACK requires only 646 short light pulses at a low frequency to achieve prolonged reduction of neuronal activity. 647 A disadvantage of PACK is its light-active component, bPAC, since it elicits side effects 648 in vivo, which are presumably related to its dark activity. In the mouse model of MTLE, 649 the light-independent effects of bPAC prevented the spread of spontaneous 650 epileptiform activity from the seizure focus to the contralateral bPAC-expressing CA1 651 region. Our study underlines that the PACK tool is a potent optogenetic inhibitor in 652 vivo but refinement of its light-sensitive domain is required to avoid dark activity and 653 related side effects.

\section{References}

655 Alfonsa H, Merricks EM, Codadu NK, Cunningham MO, Deisseroth K, Racca C, 656 Trevelyan AJ (2015) The contribution of raised intraneuronal chloride to epileptic network activity. J Neurosci 35:7715-7726.

Antoni FA (2012) New paradigms in cAMP signalling. Mol Cell Endocrinol 353:3-9. 
662 Banghart M, Borges K, Isacoff E, Trauner D, Kramer RH (2004) Light-activated ion channels for remote control of neuronal firing. Nat Neurosci 7:1381-1386.

Beck S, Yu-Strzelczyk J, Pauls D, Constantin OM, Gee CE, Ehmann N, Kittel RJ, Nagel G, Gao S (2018) Synthetic light-activated ion channels for optogenetic activation and inhibition. Front Neurosci 12:643.

Bender RA, Soleymani S V., Brewster AL, Nguyen ST, Beck H, Mathern GW, Baram TZ (2003) Enhanced expression of a specific hyperpolarization-activated cyclic nucleotide-gated cation channel $(\mathrm{HCN})$ in surviving dentate gyrus granule cells of human and experimental epileptic hippocampus. J Neurosci 23:6826-6836.

Bernal Sierra YA, Rost BR, Pofahl M, Fernandes AM, Kopton RA, Moser S, Holtkamp kainate injection in adult mice: Electroencephalography, histopathology and synaptic reorganization similar to mesial temporal lobe epilepsy. Neuroscience 89:717-729. $\mathrm{Hz}$ ) oscillation in the hippocampus of the behaving rat. J Neurosci 15:47-60.

Brams M, Kusch J, Spurny R, Benndorf K, Ulens C (2014) Family of prokaryote cyclic nucleotide-modulated ion channels. Proc Natl Acad Sci USA 111:7855-7860. network patterns of activity in the mouse. Neuroscience 116:201-211. 
unfamiliar environment. Physiol Behav 47:749-753.

Cheng X, Ji Z, Tsalkova T, Mei F (2008) Epac and PKA: A tale of two intracellular cAMP receptors. Acta Biochim Biophys Sin (Shanghai) 40:651-662.

691 Choi YS, Lee B, Hansen KF, Aten S, Horning P, Wheaton KL, Impey S, Hoyt KR, Obrietan K (2016) Status epilepticus stimulates NDEL1 expression via the CREB/CRE pathway in the adult mouse brain. Neuroscience 331:1-12.

Colgin LL (2016) Rhythms of the hippocampal network. Nat Rev Neurosci 17:239_ 249.

Conte G, Parras A, Alves M, Ollà I, De Diego-Garcia L, Beamer E, Alalqam R,

Cosentino C, Alberio L, Gazzarrini S, Aquila M, Romano E, Cermenati S, Zuccolini P, 348:707-710.

Esteller R, Echauz J, Tcheng T (2004) Comparison of line length feature before and

Engel J. J (2001) Mesial temporal lobe epilepsy: What have we learned?

Feng G, Mellor RH, Bernstein M, Keller-Peck C, Nguyen QT, Wallace M, Nerbonne JM, Lichtman JW, Sanes JR (2000) Imaging neuronal subsets in transgenic mice expressing multiple spectral variants of GFP. Neuron 28:41-51.

712 Fuhrmann F, Justus D, Sosulina L, Kaneko H, Beutel T, Friedrichs D, Schoch S, 
the speed-correlated firing of hippocampal neurons are controlled by a medial septal glutamatergic circuit. Neuron 86:1253-1264.

Fukaya R, Maglione M, Sigrist SJ, Sakaba T (2021) Rapid Ca2+ channel accumulation contributes to CAMP-mediated increase in transmission at hippocampal mossy fiber synapses. Proc Natl Acad Sci USA 118: e2016754118.

Gloor P, Salanova V, Olivier A, Quesney LF (1993) The human dorsal hippocampal commissure: An anatomically identifiable and functional pathway. Brain 116:1249-1273.

Gridchyn I, Schoenenberger P, O’neill J, Csicsvari J (2020) Optogenetic inhibitionmediated activity-dependent modification of CA1 pyramidal-interneuron connections during behavior. Elife 9:e61106.

Gruart A, Benito E, Delgado-García JM, Barco A (2012) Enhanced cAMP response element-binding protein activity increases neuronal excitability, hippocampal long-term potentiation, and classical eyeblink conditioning in alert behaving mice. J Neurosci 32:17431-17441.

Hangya B, Borhegyi Z, Szilágyi N, Freund TF, Varga V (2009) GABAergic neurons of the medial septum lead the hippocampal network during theta activity. $\mathrm{J}$ Neurosci 29:8094-8102.

Hansen KF, Sakamoto K, Pelz C, Impey S, Obrietan K (2014) Profiling status epilepticus-induced changes in hippocampal RNA expression using highthroughput RNA sequencing. Sci Rep 4: 6930.

Häussler U, Bielefeld L, Froriep UP, Wolfart J, Haas CA (2012) Septotemporal position in the hippocampal formation determines epileptic and neurogenic activity in temporal lobe epilepsy. Cereb Cortex 22:26-36.

He C, Chen F, Li B, Hu Z (2014) Neurophysiology of HCN channels: From cellular functions to multiple regulations. Prog Neurobiol 112:1-23. 
740 Heining K, Kilias A, Janz P, Häussler U, Kumar A, Haas CA, Egert U (2019) Bursts with high and low load of epileptiform spikes show context-dependent correlations in epileptic mice. eNeuro 6:ENEURO.0299-18.2019.

Heinrich C, Nitta N, Flubacher A, Müller M, Fahrner A, Kirsch M, Freiman T, Suzuki F, Depaulis A, Frotscher M, Haas CA (2006) Reelin deficiency and displacement of mature neurons, but not neurogenesis, underlie the formation of granule cell dispersion in the epileptic hippocampus. J Neurosci 26:4701-4713.

Henß T, Nagpal J, Gao S, Scheib U, Pieragnolo A, Hirschhäuser A, Schneidermanipulation of cyclic nucleotides functionally coupled to cyclic nucleotide-gated channels. Br J Pharmacol: 10.1111/bph.15445.

Hinman JR, Penley SC, Long LL, Escabí MA, Chrobak JJ (2011) Septotemporal variation in dynamics of theta: Speed and habituation. J Neurophysiol 105:2675-

Huang YY, Li XC, Kandel ER (1994) cAMP contributes to mossy fiber LTP by initiating both a covalently mediated early phase and macromolecular synthesispotassium-selective glutamate receptor for the optical inhibition of neuronal firing. Nat Neurosci 13:1027-1032. 
Cereb Cortex 27:2348-2364.

Jiang Y, Gavrilovici C, Chansard M, Liu RH, Kiroski I, Parsons K, Park SK, Teskey

Kang JY, Kawaguchi D, Coin I, Xiang Z, O’Leary DDM, Slesinger PA, Wang L (2013)

Kwan P, Sander JW (2004) The natural history of epilepsy: An epidemioloqical view. J Neurol Neurosurg Psychiatry 75:1376-1381.

Lewis AS, Chetkovich DM (2011) HCN channels in behavior and neurological disease: Too hyper or not active enough? Mol Cell Neurosci 46:357-367. compartment-dependent distribution of HCN1 in pyramidal cell dendrites. Nat Neurosci 5:1185-1193.

Mahn M, Prigge M, Ron S, Levy R, Yizhar O (2016) Biophysical constraints of optogenetic inhibition at presynaptic terminals. Nat Neurosci 19:554-556.

Mattis J, Tye KM, Ferenczi EA, Ramakrishnan C, O'Shea DJ, Prakash R, Gunaydin LA, Hyun M, Fenno LE, Gradinaru V, Yizhar O, Deisseroth K (2012) Principles for applying optogenetic tools derived from direct comparative analysis of microbial opsins. Nat Methods 9:159-172.

Meier R, Häussler U, Aertsen A, Deransart C, Depaulis A, Egert U (2007) Short-term 1040.e3. 
Mintzer S, Cendes F, Soss J, Andermann F, Engel J, Dubeau F, Olivier A, Fried I (2004) Unilateral hippocampal sclerosis with contralateral temporal scalp ictal onset. Epilepsia 45:792-802.

Müller C, Remy S (2018) Septo - hippocampal interaction. Cell Tissue Res 373:565575.

Nguyen P V., Woo NH (2003) Regulation of hippocampal synaptic plasticity by cyclic AMP-dependent protein kinases. Prog Neurobiol 71:401-437.

Noam Y, Bernard C, Baram TZ (2011) Towards an integrated view of HCN channel role in epilepsy. Curr Opin Neurobiol 21:873-879.

Nolan M, Malleret G, Dudman J, Buhl D, Santoro B, Gibbs E, Vronskaya S, Buzsaki G, Siegelbaum S, Kandel E (2004) A behavioral role for dendritic integration: HCN1 channels constrain spatial memory and plasticity at inputs to distal BR (2021) SynaptoPAC, an optogenetic tool for induction of presynaptic plasticity. J Neurochem 156:324-336.

Owen SF, Liu MH, Kreitzer AC (2019) Thermal constraints on in vivo optogenetic

$$
\text { M, Egert U, Janz P, Haas CA (2020) Hippocampal low-frequency stimulation }
$$

Popovic L, Vojvodic N, Ristic AJ, Bascarevic V, Sokic D, Kostic VS (2012) Ictal prevents seizure generation in a mouse model of mesial temporal lobe epilepsy. dystonia and secondary generalization in temporal lobe seizures: A video-EEG

817 Racine RJ (1972) Modification of seizure activity by electrical stimulation. II. Motor 
seizure. Electroencephalogr Clin Neurophysiol 32:281-294.

819 Raimondo J V., Kay L, Ellender TJ, Akerman CJ (2012) Optogenetic silencing strategies differ in their effects on inhibitory synaptic transmission. Nat Neurosci 15:1102-1104.

Rangel LM, Rueckemann JW, Riviere PD, Keefe KR, Porter BS, Heimbuch IS, Budlong $\mathrm{CH}$, Eichenbaum H (2016) Rhythmic coordination of hippocampal neurons during associative memory processing. Elife 5:1-24.

Riban V, Bouilleret V, Pham-Lê BT, Fritschy JM, Marescaux C, Depaulis A (2002) hippocampal sclerosis in a mouse model of temporal lobe epilepsy.

Santoro B, Chen S, Lüthi A, Pavlidis P, Shumyatsky GP, Tibbs GR, Siegelbaum SA (2000) Molecular and functional heterogeneity of hyperpolarization-activated

Scheib U, Broser M, Constantin OM, Yang S, Gao S, Mukherjee S, Stehfest K, Nagel

$$
\text { G, Gee CE, Hegemann P (2018) Rhodopsin-cyclases for photocontrol of }
$$
cGMP/cAMP and $2.3 \AA$ structure of the adenylyl cyclase domain. Nat Commun

Sørensen AT, Ledri M, Melis M, Ledri LN, Andersson M, Kokaia M (2017) Altered chloride homeostasis decreases the action potential threshold and increases lobe epilepsy. Cereb Cortex 22:2087-2101. 
modulation of cellular cAMP by a small bacterial photoactivated adenylyl cyclase, bPAC, of the soil bacterium Beggiatoa. J Biol Chem 286:1181-1188.

Tukker JJ, Fuentealba P, Hartwich K, Somogyi P, Klausberger T (2007) Cell typespecific tuning of hippocampal interneuron firing during gamma oscillations in vivo. J Neurosci 27:8184-8189.

Tulke S, Haas CA, Häussler U (2019) Expression of brain-derived neurotrophic factor and structural plasticity in the dentate gyrus and CA2 region correlate with epileptiform activity. Epilepsia 60:1234-1247.

Twele F, Schidlitzki A, Töllner K, Löscher W (2017) The intrahippocampal kainate mouse model of mesial temporal lobe epilepsy: Lack of electrographic seizurelike events in sham controls. Epilepsia Open 2:180-187.

Vaden JH, Banumurthy G, Gusarevich ES, Overstreet-Wadiche L, Wadiche JI (2019) The readily-releasable pool dynamically regulates multivesicular release. Elife 8:

Weisskopf MG, Castillo PE, Zalutsky RA, Nicoll RA (1994) Mediation of Hippocampal Mossy Fiber Long-Term Potentiation by Cyclic AMP. Science 265:1878-1882.

Wiegert JS, Mahn M, Prigge M, Printz Y, Yizhar O (2017) Silencing Neurons: Tools, Applications, and Experimental Constraints. Neuron 95:504-529.

Wozny C, Maier N, Fidzinski P, Breustedt J, Behr J, Schmitz D (2008) Differential cAMP signaling at hippocampal output synapses. J Neurosci 28:14358-14362.

Zhu L, Dai S, Lu D, Xu P, Chen L, Han Y, Zhong L, Chang L, Wu Q (2020) Role of NDEL1 and VEGF/VEGFR-2 in mouse hippocampus after status epilepticus. ASN Neuro 12:1759091420926836. 


\section{Figures}

A

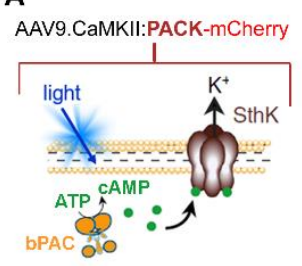

AAV9.CaMKII:PACK-mCherry

AAV injection
C

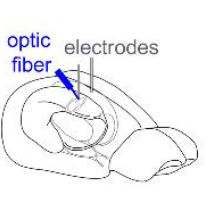

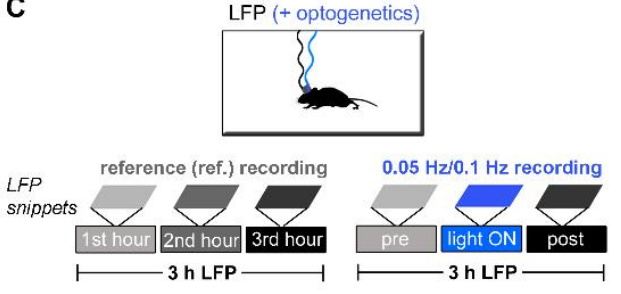

D

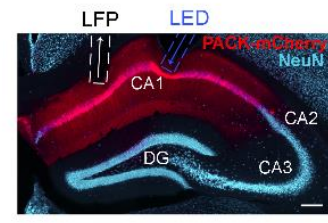

E

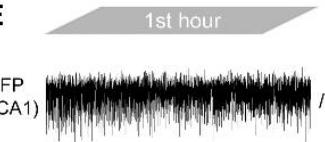

G

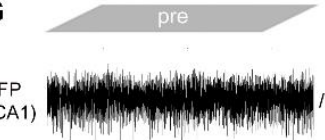

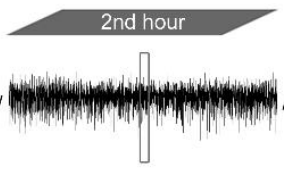
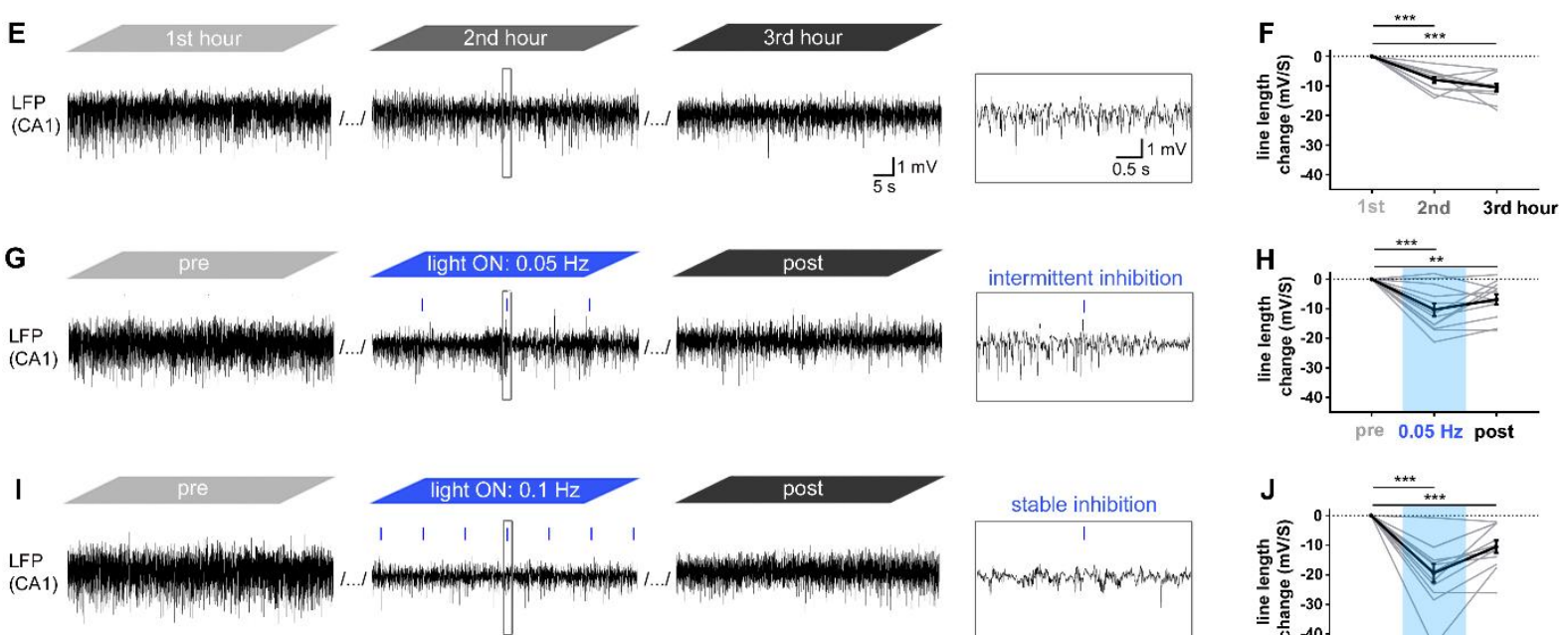

Figure 1. PACK-mediated optogenetic inhibition of CA1 principal cells in vivo. (A-D) Experimental design with a timeline. (A) We targeted PACK (bPAC+Sthk) to excitatory neurons by injecting the AAV9.CaMKII vector into the CA1 region of the hippocampus.

(B) After implantations of wire electrodes and optic fibers into CA1,
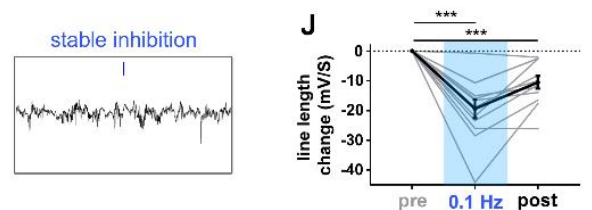
(ref.) recordings were without illumination, whereas the ' $0.05 \mathrm{~Hz} / 0.1 \mathrm{~Hz}$ recordings' included one-hour pre-recording, light ON phase with $0.05 \mathrm{~Hz}$ or $0.1 \mathrm{~Hz}$ illumination, and a post-recording. Each recording type (ref., $0.05 \mathrm{~Hz}, 0.1 \mathrm{~Hz}$ ) was performed twice in each mouse (for details see Materials and Methods). (D) PACK-mCherry expression and electrode/optic fiber positions in CA1 were confirmed by histology at the end of the experiment. (E, G, I) Representative LFP snippets from the first, second, and third hour of each recording type. (E) In ref. recordings, LFP magnitude decreased over time, (F) confirmed by the significant drop of mean line length (in black) in the second and third recording hour. (G) Applying $10 \mathrm{~ms}$ blue light pulses $\left(\sim 80 \mathrm{~mW} / \mathrm{mm}^{2}, 460 \mathrm{~nm}\right)$ at $0.05 \mathrm{~Hz}$ resulted in an intermittent reduction of LFP magnitude. $(\mathrm{H})$ Mean line length was significantly reduced during $0.05 \mathrm{~Hz}$ light application and in the post-recording. (I) Applying light pulses at $0.1 \mathrm{~Hz}$ resulted in a stable reduction of LFP magnitude, (J) which is reflected in a robust reduction of line length during $0.1 \mathrm{~Hz}$ illumination. One-sample t-test $(n=12$ trials from 6 mice, grey lines), ${ }^{* *} p<0.01,{ }^{* * *} p<0.001$. Mean presented in black with SEM as error bars. 
A

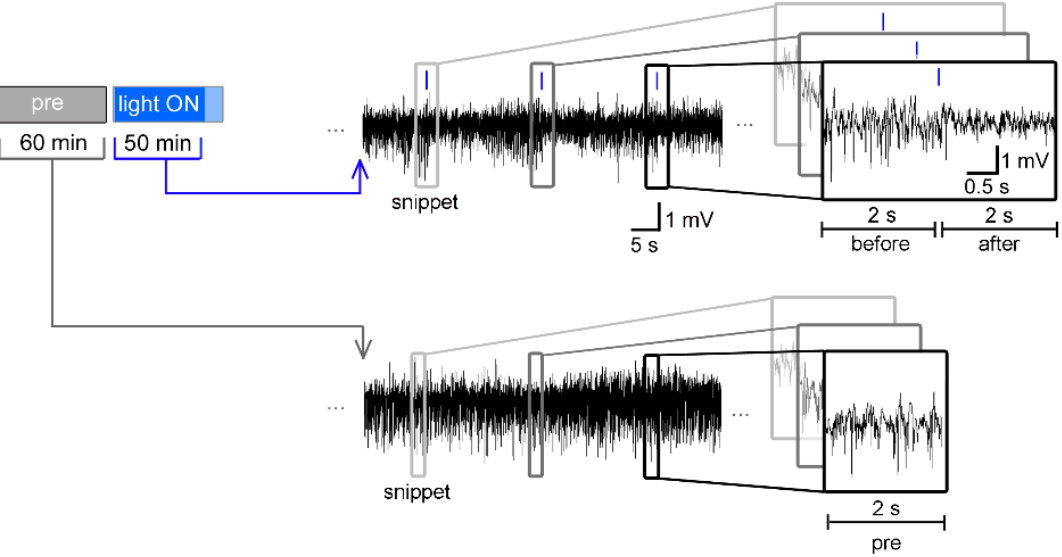

B

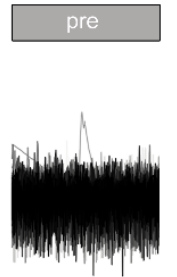

D

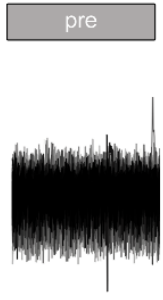

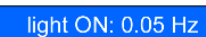

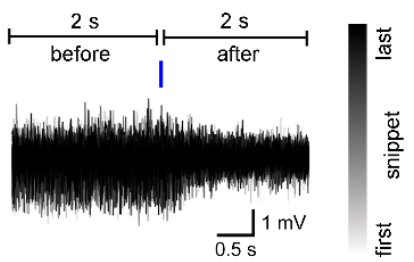

light $\mathrm{ON}: 0.1 \mathrm{~Hz}$

I

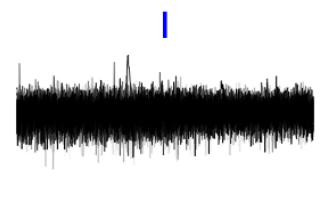

C intermittent inhibition

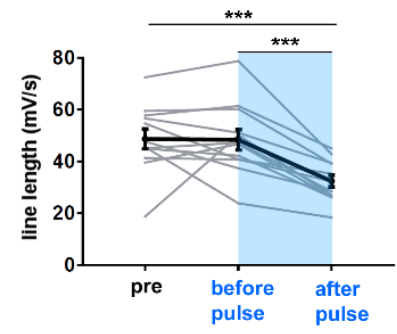

E

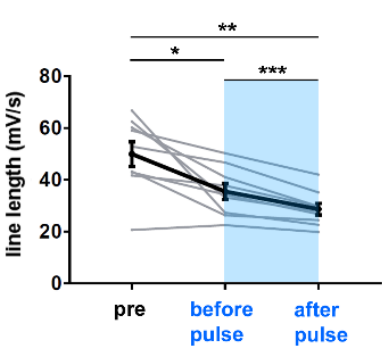

Figure 2. PACK-mediated inhibition in vivo is reliable and reversible. (A) Analysis of responses to $10 \mathrm{~ms}$ blue light pulses during the first 50 minutes of the light ON phase. Two seconds long LFP snippets before and after each light pulse were extracted and overlaid with a color-coding from grey to black (first to last LFP snippet). To enable comparison to baseline two-second snippets at corresponding time points were extracted from pre-recordings. (B, D) Representative plots of overlaid LFP snippets from a pre-recording and light $\mathrm{ON}$ recording with (B) $0.05 \mathrm{~Hz}$ and

(D) $0.1 \mathrm{~Hz}$ illumination. $(C, E)$ Line lengths

893 ( $\mathrm{mV} / \mathrm{s})$ were calculated for each two-second LFP snippet and the mean "pre", "before pulse", and "after pulse" line length for each recording $(n=12)$ is presented (in grey). The mean (in black) "after pulse" line length was significantly smaller than "pre" and "before pulse" line length in both illumination modes, indicating a reliable reduction of neuronal activity. (C) Baseline ("pre") and "before pulse" line lengths were similar in $0.05 \mathrm{~Hz}$ sessions, demonstrating recovery from inhibition before the next pulse was applied. (E) LFP line length was reduced throughout the $0.1 \mathrm{~Hz}$ light ON period (in blue), including before and after pulse phases, suggesting the inhibition was stable. RM ANOVA and Tukey's multiple comparisons test ( $n=12$ trials from 6 mice, grey lines), ${ }^{*} p<0.05,{ }^{* *} p<0.01,{ }^{* * *} p<0.001$. Mean presented in black with SEM as error bars. 
A
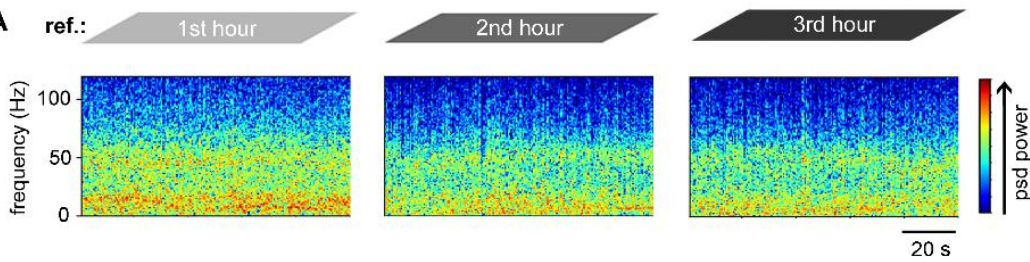

C $0.1 \mathrm{~Hz}$
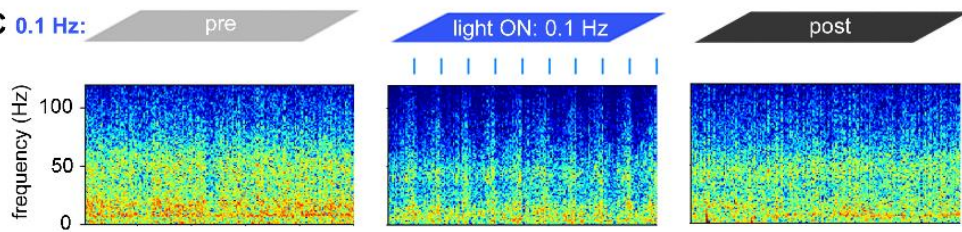

E

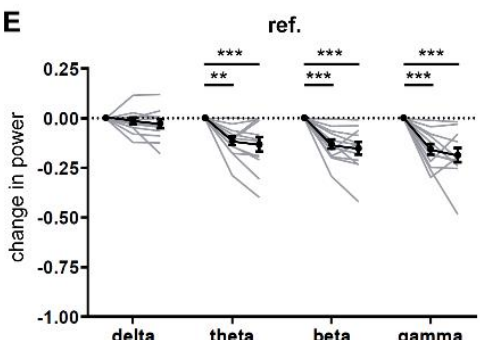

$\mathbf{F}$

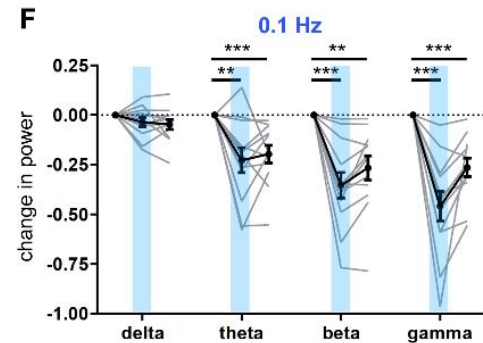

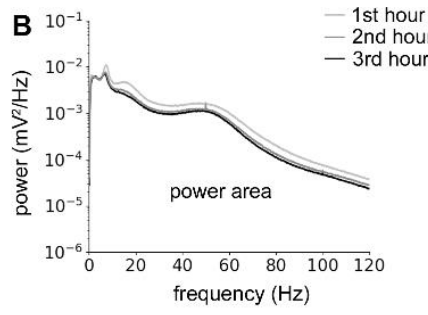

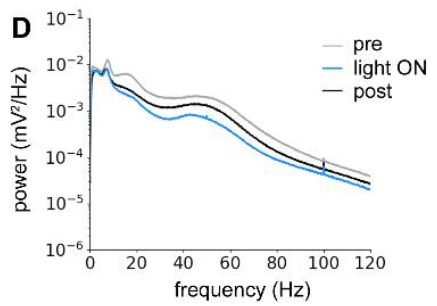

G

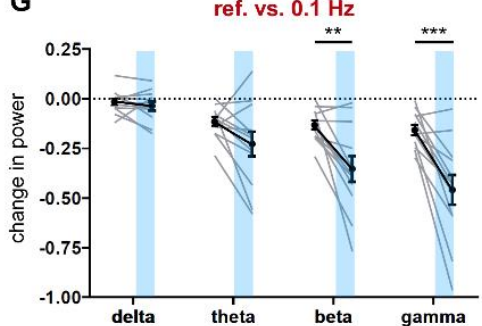

Figure 3. Spectral analysis reveals reduced power of beta and gamma oscillations during lightactivation of PACK. (A) Representative spectrogram snippets from a ref. recording show baseline activity; blue colors reflecting low power and red colors reflecting high power of the corresponding frequencies. (B) Mean power spectral density (PSD) during the first (light grey), second (dark grey), and third (black) recording hour. (C) Light pulses applied at $0.1 \mathrm{~Hz}$ transiently decreased the spectral power.

908 (D) The mean PSDs from pre- (light grey), light ON (blue), and post-recordings (black). (E-G) The 909 oscillatory power was calculated by taking the AUC in the PSD plot for the particular frequency range.

910 The power change is presented as baseline corrected values for the second and third hour. During the 911 (E) ref. and $(F)$ illumination recordings with $0.1 \mathrm{~Hz}$, the power of theta, beta, and gamma declined 912 significantly. $(G)$ Change in power in the second hour of ref. versus $0.1 \mathrm{~Hz}$ recordings revealed that only 913 beta and gamma power were further reduced by the light-induced PACK activation. 
A

AAV9.CaMKII:bPAC-mCherry

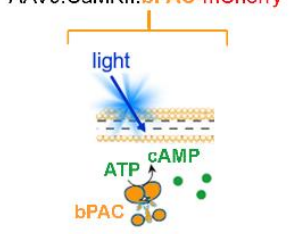

AAV injection

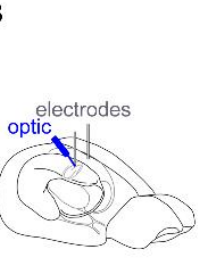

C
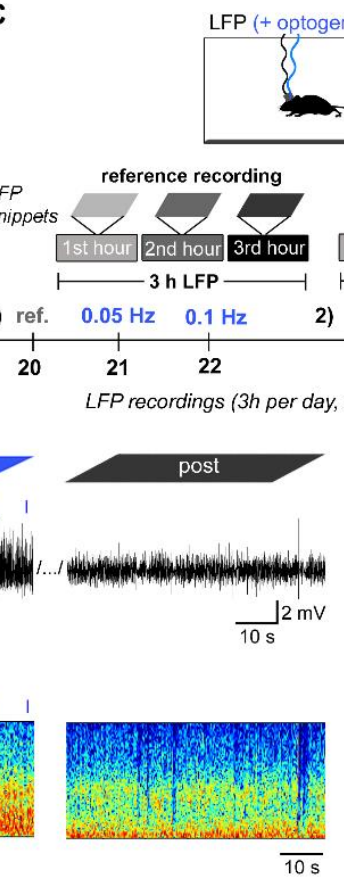

914

915

916

917

918

919

920

921

922

923

924

925

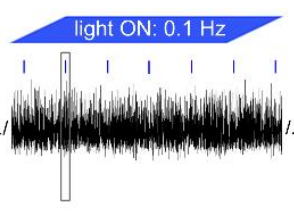

G
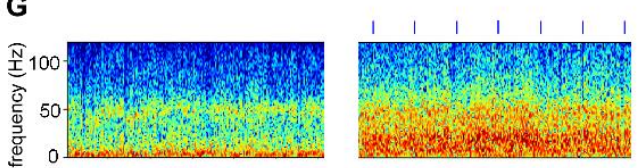

Figure 4. Light-activation of bPAC reversibly increases neuronal activity in CA1. (A) Experimental design. We targeted bPAC-mCherry to excitatory neurons in the CA1 area using the AAV9 vector. (B) Implantation, (C) recordings and (D) histological analysis were performed like in PACK mice. Scale bar $200 \mu \mathrm{m}$. (E) Representative snippets of a $0.1 \mathrm{~Hz}$ recording show persistently increased LFP magnitude during the light ON phase in bPAC-expressing CA1. (F) In the light ON phase, the mean line length was significantly increased compared to the baseline (pre) level. In the post-recordings, the line length was reduced compared to the baseline. $(G)$ The spectrograms, taken from the same time window as LFP snippets, demonstrate elevated spectral power. $(\mathrm{H})$ The mean PSD during $0.1 \mathrm{~Hz}$ illumination was increased at frequencies above $\sim 10 \mathrm{~Hz}$. (I) The beta and gamma powers were significantly higher during the light $\mathrm{ON}$ phase compared to the pre-level. One-sample t-test ( $\mathrm{n}=12$ trials from 6 mice, grey lines), ${ }^{*} p<0.05,{ }^{* *} p<0.01$. Mean presented in black with SEM as error bars. 
A

Spontaneous GS in a PACK mouse

right $\mathrm{HC}$ (PACK)
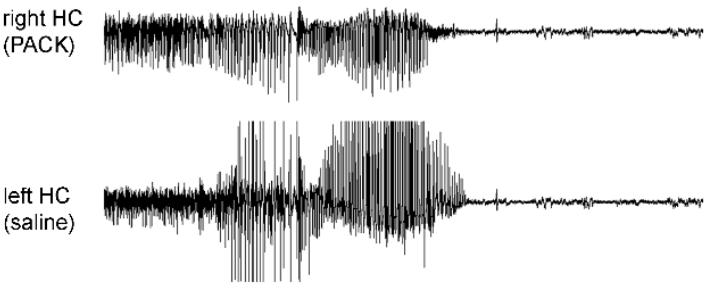

C

Occurrence of GS in PACK mice

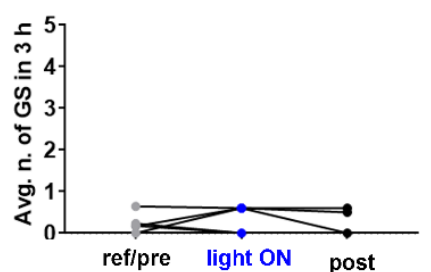

D Occurrence of GS in bPAC mice

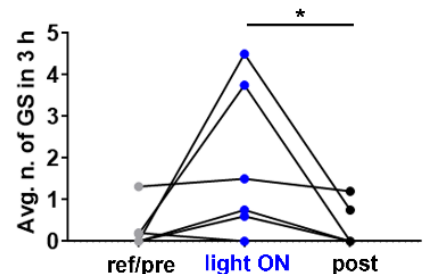

Spontaneous GS in a bPAC mouse

left $\mathrm{HC}$

(saline)

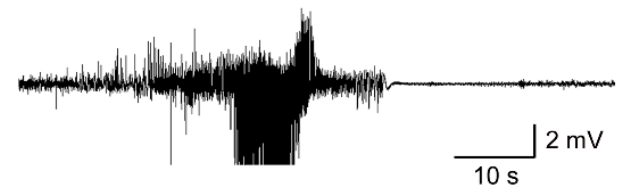

E

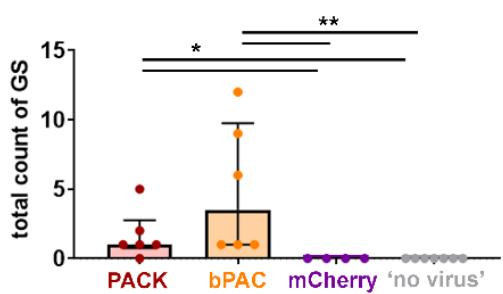

Figure 5. Spontaneous generalized seizures (GS) in PACK and bPAC mice during LFP recordings.

(A) Representative spontaneous electrographic GS in a PACK mouse. The hypersynchronous activity spread across both hemispheres and was followed by postictal depression. (B) Similar GS were recorded in bPAC mice. (C,D) The number of GS was counted in all LFP recording types (baseline: "ref/pre", "light ON", "post") of PACK ( $n=6)$ and bPAC $(n=6)$ mice, and the average number of GS in three

932 hours is presented and compared with Friedman's test followed by Dunn's multiple comparisons. The

933 occurrence of GS in baseline recordings indicates seizure generalization independent from light-induced PACK/bPAC activation. (E) The total count of GS in LFP recordings (9-28 h) was the highest in bPAC mice. Control mice that expressed either mCherry in CA1 or no virus, experienced no seizure-like activity during recordings. Kruskal-Wallis test with Dunn's multiple comparisons, ${ }^{*} p<0.05,{ }^{* *} p<0.05$. Median presented with IQR as error bars. 

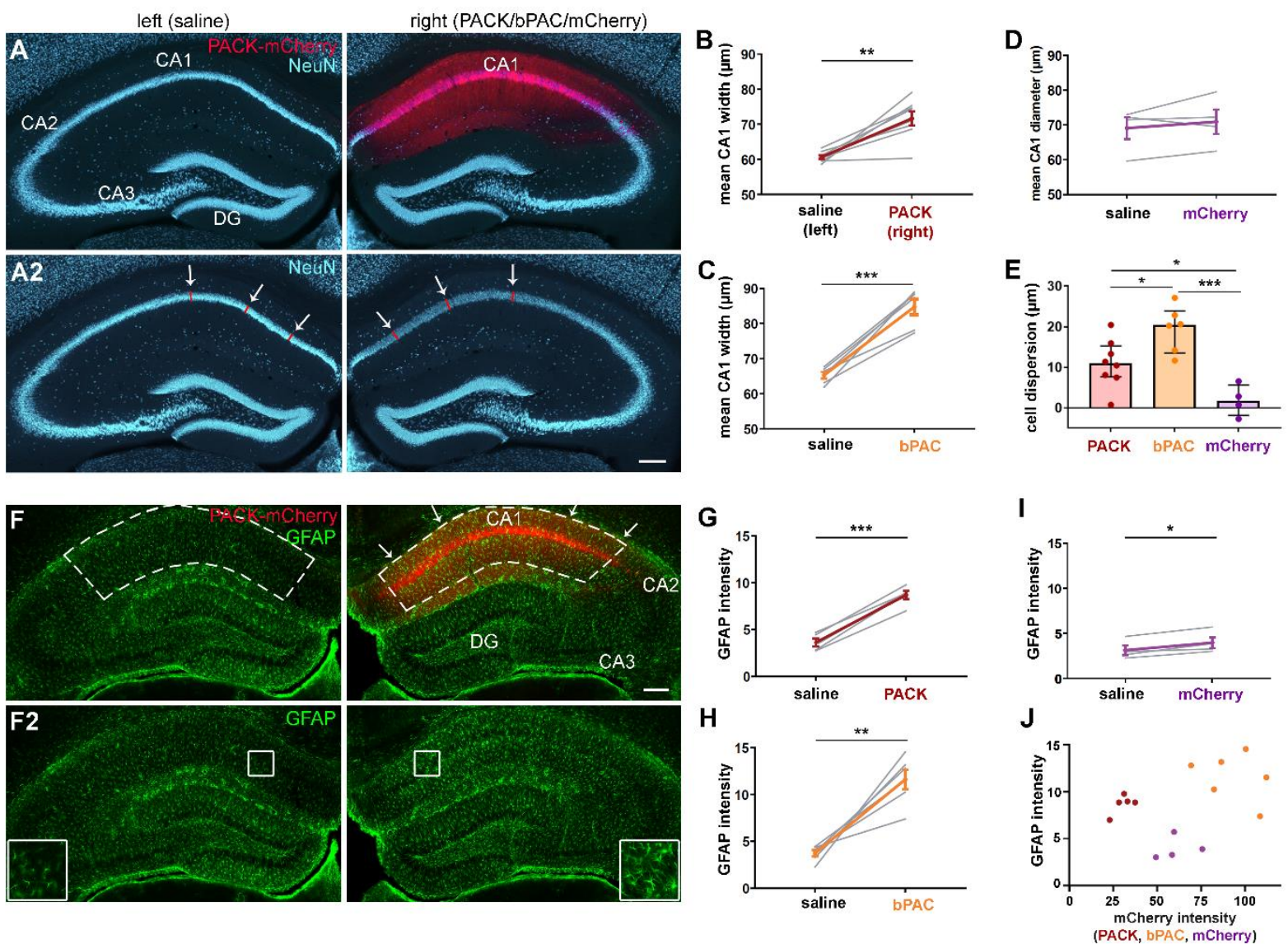

Figure 6. Cell dispersion and astrogliosis in PACK- and bPAC-expressing CA1. (A) Representative image of a NeuN-labeled hippocampal section with PACK-mCherry expression in the right CA1. The diameter of the CA1 pyramidal cell layer was measured at six positions (red lines and white arrows) in three hippocampal sections per animal. $(B, C)$ The mean width of the CA1 pyramidal cell layer in (B)

943 PACK-expressing (in red) and (C) in bPAC-expressing (in orange) hippocampus, was significantly 944 increased compared to the contralateral saline-injected hippocampus (paired t-test). (D) In mCherry945 expressing CA1, the pyramidal cell layer width was similar as in the contralateral CA1. (E) The cell 946 dispersion (right-left CA1 width) was the strongest in bPAC mice (one-way ANOVA, Tukey's post hoc).

947 (F) In the representative image of a GFAP-stained section, GFAP labeling was visibly stronger in the 948 PACK-expressing CA1. Mean grey value of GFAP labeling and mCherry expression was measured in 949 the right dorsal CA1 including str. oriens, str. pyramidale, and str. radiatum (white dashed lines with 950 arrows) in three sections per animal. GFAP intensity was measured at same positions in the left CA1 951 (white dashed lines). (G, H) GFAP labeling intensity was significantly higher in (G) PACK, (H) bPAC, and 952 (I) mCherry-expressing CA1 compared to the left saline-injected side (paired t-test). (J) There was no 953 clear correlation between GFAP labeling intensity and mCherry intensity, however PACK, bPAC, and 954 mCherry mice formed three separate clusters with mCherry mice having the lowest GFAP labeling. $955{ }^{*} p<0.05,{ }^{* *} p<0.01,{ }^{* * *} p<0.001$. Mean presented with SEM as error bars. Scale bars $200 \mu \mathrm{m}$. 


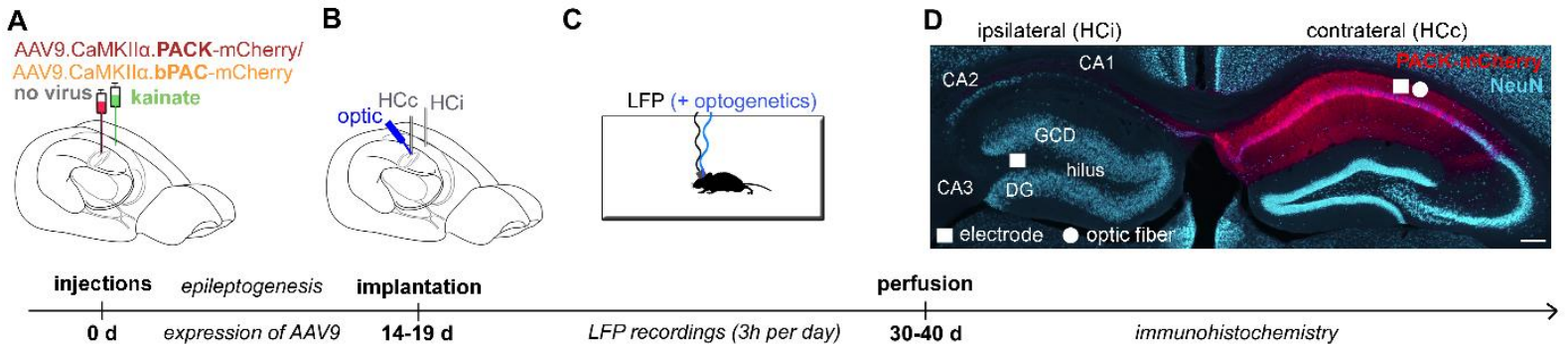

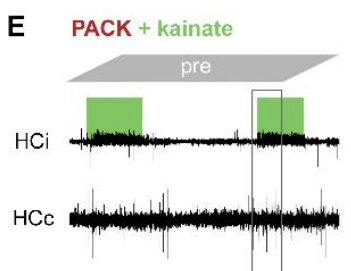

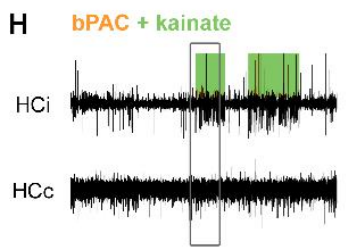

K

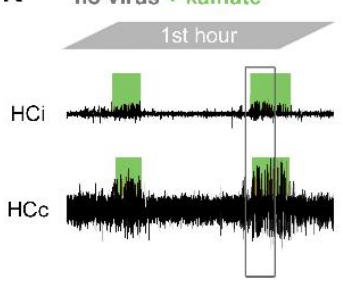

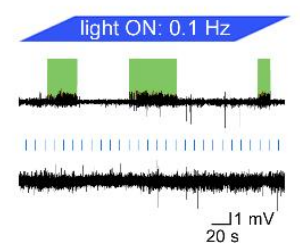
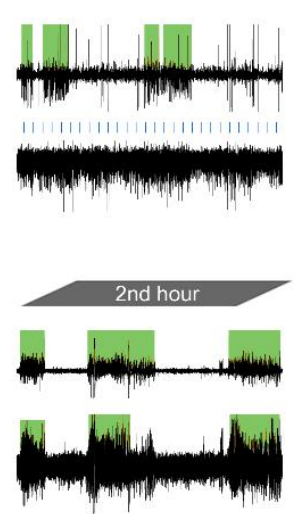
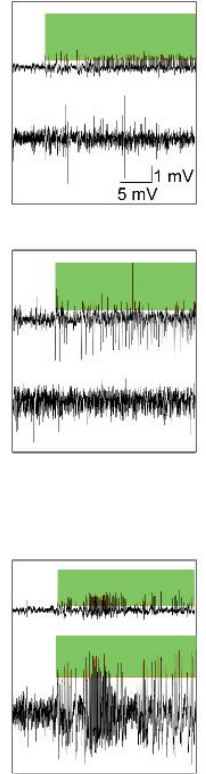
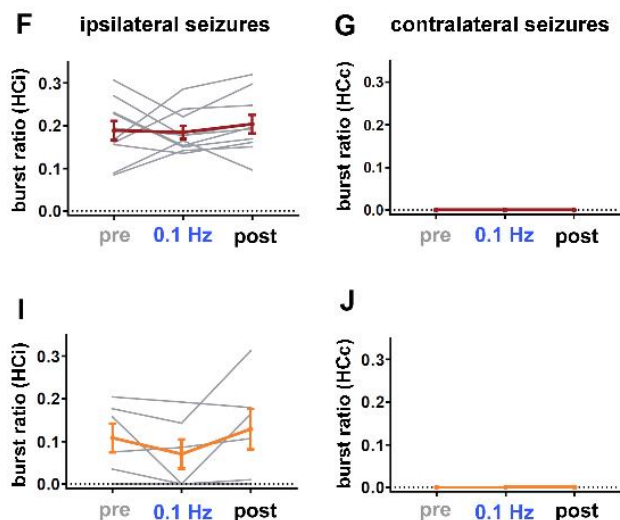

L

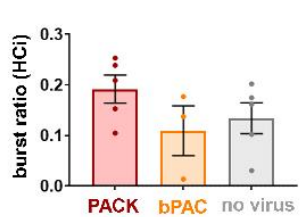

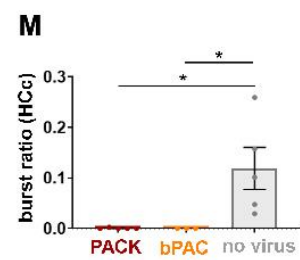

956

957

958

959

960

961

962

963

964

965

966

967

968

969

970

971

972

973

974

975

Figure 7. Chronically epileptic PACK and bPAC mice lack spontaneous seizures in the contralateral hippocampus. (A) Mice were injected with kainate in the left hippocampus and AAV9 carrying PACK, bPAC, or no virus in the contralateral hippocampus. (B) Two weeks later, wire electrodes were implanted into the kainate-injected ipsilateral hippocampus $(\mathrm{HCi})$ and the virus-injected contralateral hippocampus (HCc). In PACK and bPAC mice, an optic fiber was implanted at a $30^{\circ}$ angle adjacent to HCc. (C) Three-hour LFP recordings with and without optogenetic manipulations were performed as previously in healthy PACK/bPAC mice. 'No virus' mice were recorded only for three-hour reference recordings. (D) Representative section of an ihpKA PACK mouse showing hippocampal sclerosis in $\mathrm{HCi}$ with cell loss in CA1, CA3, and hilus regions as well as granule cell dispersion (GCD). PACK-mCherry was expressed in dorsal CA1 of HCc. Representative LFP snippets from (E) PACKexpressing $(\mathrm{H})$ bPAC-expressing and $(\mathrm{K})$ 'no virus' kainate-injected mice in the chronic phase of epilepsy. (E-M) Spontaneous epileptiform bursts (hypersynchronous spiking activity, marked in green) were detected by an automated algorithm (Heining et al., 2019), and quantified as burst ratio, a fraction of recording spent in bursts. (E-G) PACK and (H-J) bPAC mice had regularly occurring seizures in $\mathrm{HCi}$ but no propagation to HCc during pre-recordings as well as during and after illumination. (K) In contrast, mice without virus expression, frequently showed seizure propagation to HCc. (L) The burst ratio in $\mathrm{HCi}$ was similar in PACK, bPAC, and 'no virus' mice, (M) whereas in $\mathrm{HCc}$, the burst ratio was significantly above zero in 'no virus' epileptic mice (33-36 days after inpKA). One-way ANOVA with Dunnett's multiple comparison test, ${ }^{*} \mathrm{p}<0.05$. Mean presented with SEM as error bars. 


\section{Table}

977 Table 1. Experimental groups and sample sizes in LFP analysis. From the initial number of mice

978 that entered the experiment (total injected), some died owing to intrahippocampal kainate injections or 979 implantations, and some were excluded due to electrode/optic fiber positions not in CA1 or due to 980 hippocampal atrophy. The sample size indicates the number of mice included in LFP analysis.

\begin{tabular}{l|cccc} 
Group & Total injected & Died due to surgery & Excluded & LFP analysis \\
\hline saline + PACK & 12 & 2 & 4 & 6 \\
saline + bPAC & 7 & 1 & 0 & 6 \\
saline + mCherry & 4 & 1 & 1 & 3 \\
saline & 7 & 0 & 0 & 7 \\
kainate + PACK & 11 & 4 & 2 & 5 \\
kainate + bPAC & 9 & 6 & 0 & 3 \\
kainate & 4 & 0 & 0 & 4
\end{tabular}

\section{Supplementary data}
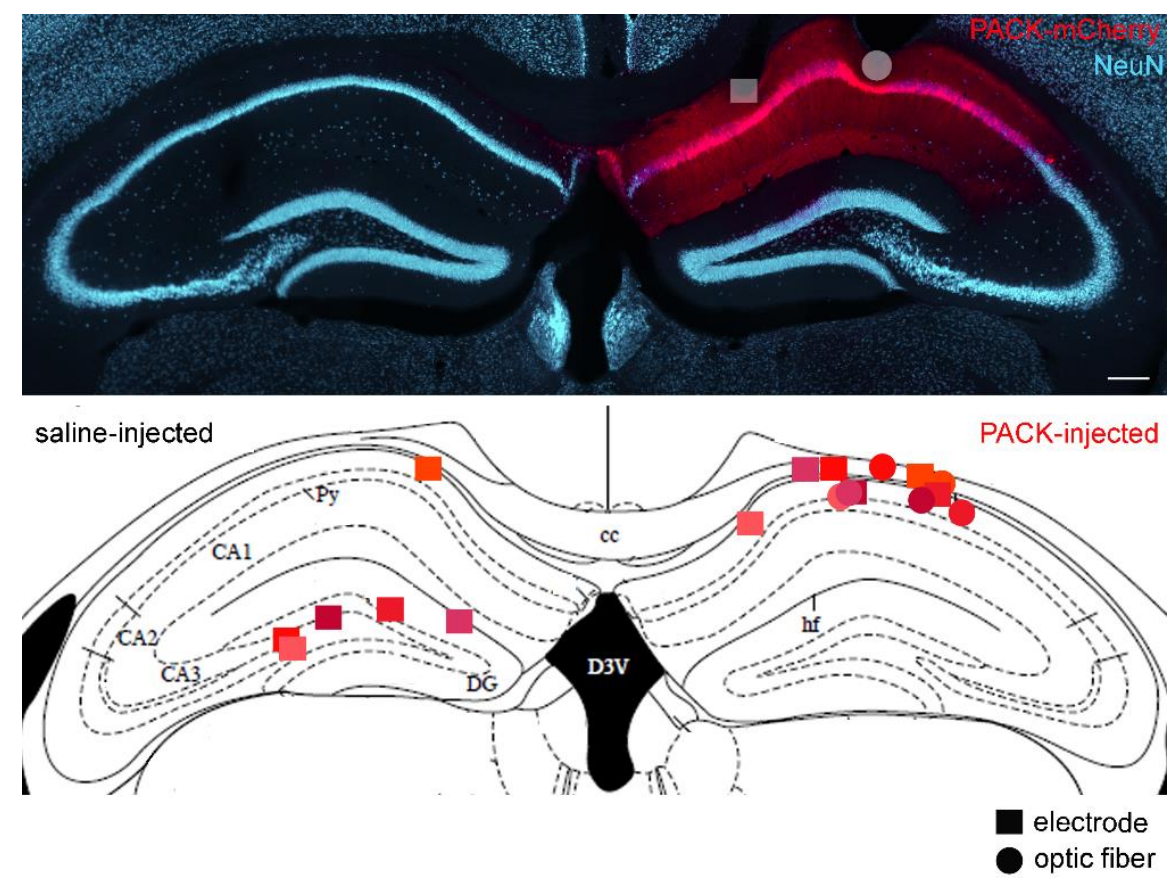

Supplementary figure 1. Electrode and optic fiber positions in dorsal hippocampi of PACK mice.

984 (A) A representative NeuN-labeled hippocampal section, where the electrode (square) and optic fiber

985 (circle) positions are visible in PACK-expressing right hippocampus. (B) Electrode and optic fiber 986 positions in saline-injected and PACK-injected hippocampi of mice that were included in the LFP analysis $987(n=6$, in different shades of red $)$. 

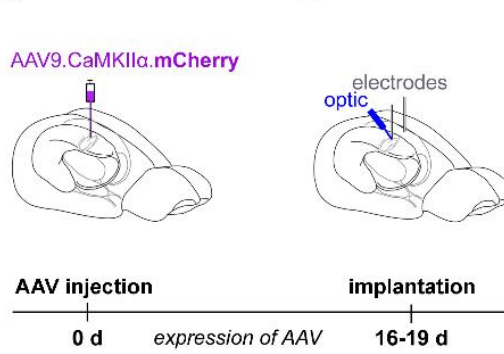

C

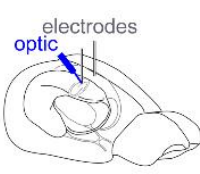

LFP
snippets

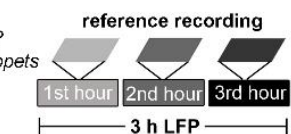

LFP (+ optogenetics)

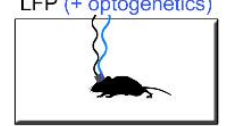

$0.05 \mathrm{~Hz} / 0.1 \mathrm{~Hz}$ recording

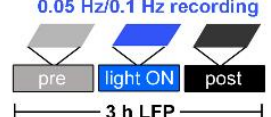

$\begin{array}{lllllll}20 & 21 & 22 & 25 & 26 & 27 & 30-35 \mathrm{~d}\end{array}$ LFP recordings (3h per day, 2 sessions)

E

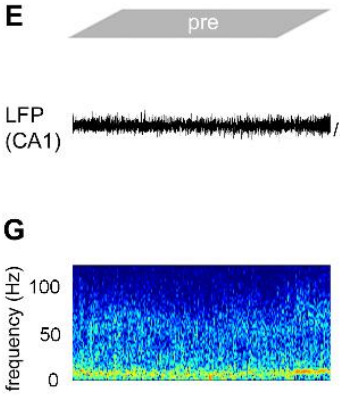

988

989

990

991

992

993

994

995

996

997

998
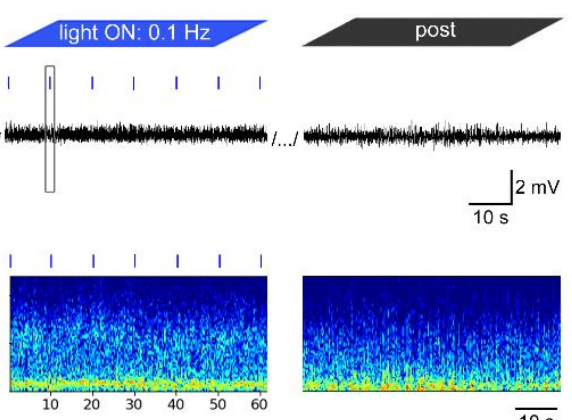

$\overline{10 s}$
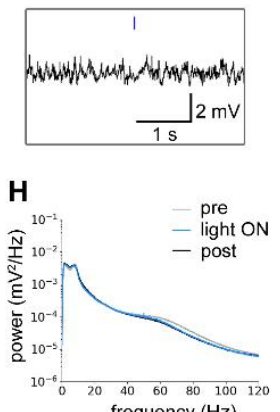

D
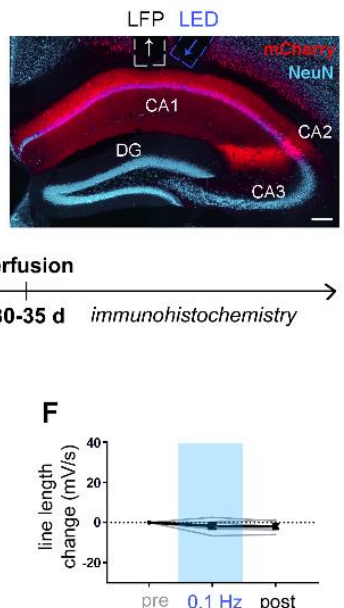

I

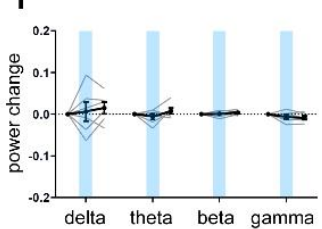

Supplementary figure 2. Light application in mCherry mice does not alter neuronal activity in

CA1. Experimental design. We targeted mCherry to excitatory neurons in the CA1 area using the AAV9 vector. (B) Implantation, (C) recordings and (D) histological analysis were performed like in PACK mice. Scale bar $200 \mu \mathrm{m}$. (E) Representative LFP snippets of a $0.1 \mathrm{~Hz}$ recording show no response to the light application. (F) Line length was not changed by $0.1 \mathrm{~Hz}$ illumination. $(G)$ The spectrograms, taken from the same time windows as LFP snippets, were unaltered during $0.1 \mathrm{~Hz}$ illumination. $(\mathrm{H})$ The average PSD is nearly overlapping during pre-, light $\mathrm{ON}$, and post-recordings, suggesting the light application did not affect the oscillations in the CA1 region. (I) The power of delta, theta, beta, and gamma oscillations remained the same across the three recording hours, not being altered by the illumination (shown in blue). 